\title{
Sampling-Type Representations of Signals and Systems
}

\author{
Holger Boche \\ Lehrstuhl für Theoretische \\ Technische Universität Munchen \\ 80290 Munchen, Germany \\ boche@tum.de \\ Ullrich J. Mönich \\ Heinrich-Hertz-Chair for Mobile Communications \\ Technische Universität Berlin \\ Einsteinufer 25 \\ D-10578 Berlin, Germany \\ ullrich.moenich@mk.tu-berlin.de
}

\begin{abstract}
In practical applications the sole reconstruction of signals by its samples is sometimes not sufficient. Often, some processed version of the signal is of interest and must be approximated by using only the samples of the signal. In this paper, the possible reconstruction kernels are characterized. Then, the convergence behavior of general approximation processes for translation invariant, linear, and bounded operators is analyzed for signals in the Paley-Wiener space $\mathcal{P} \mathcal{W}_{\pi}^{1}$ and these kernels. It is shown that the Hilbert transform is a universal operator in the sense that the peak value of all possible approximation processes diverges unboundedly for some signal in $\mathcal{P} \mathcal{W}_{\pi}^{1}$, regardless of the oversampling factor and the kernel. Furthermore, for all approximation processes and all points in time, there exists an operator such that the approximation process diverges in this point. The results are compared to the approximation behavior of the Hilbert transform, operating on continuous-time signals. Moreover, a simple criterion based on the exponential function as test signal is developed for answering the question of whether or not an approximation process is convergent for a given operator.
\end{abstract}

Key words and phrases : Sampling series, uniform approximation, reconstruction process, sampling-based signal processing, system representation 


\section{Introduction}

In many application areas the conversion of continuous-time signals to discretetime signals and vice versa plays an important role. This is due to the fact that modern signal processing is done nearly always with digital processors while the physical quantities of the real world are always analog, i.e., continuous in time. Therefore, the conversion of the discrete-time signal back into a continuous-time signal by some reconstruction process is essential. The Shannon sampling series [26] is probably the most prominent example of a reconstruction process. It has been widely used and plays an important role in many theoretical concepts. In his "Lectures on Computation," Richard Feynman discusses the problem of transmitting a function of time and writes in this context: "Consideration of such a problem will bring us on to consider the famous Sampling Theorem, another baby of Claude Shannon" [10, p. 132].

The sampling theorem has a long history and many famous names like Whittaker [29], Kotel'nikov [15], and Raabe [23] are linked with its discovery. Shannon originally introduced the sampling theorem for signals with finite energy. This special case is examined under various aspects in [28]. Many authors have tried to extend the sampling theorem to a broader class of functions, e.g., to generalized bandlimited functions [30, 7, 19]. However, these extensions consider only the pointwise convergence of the corresponding series. This means that for a given order of the partial sum, the approximation error cannot be bounded uniformly for all points in time, which complicates the use of these series in real applications. Hence, we focus on convergence that is uniform and the signal space $\mathcal{P} \mathcal{W}_{\pi}^{1}$, which is the largest space in the scale of Paley-Wiener spaces.

Sampling series with more general kernels (bandlimited and non-bandlimited) have been analyzed in numerous publications. [13] deals with finite-energy bandlimited signals and gives an upper bound for the truncation error of the Shannon sampling series with oversampling. The truncation error for nonbandlimited, uniformly continuous and bounded signals is studied in [6]. Further results can be found in [12, Chapter 6] and [4, 5, 9]. Sampling series with Gaussian multipliers are another topic, which recently attracted attention. In [21] an upper bound for the peak approximation error of the Shannon sampling series with Gaussian multiplier was derived for certain bandlimited signals with finite energy. [25] extends the result by providing error bounds for larger signal spaces, including non-bandlimited signals and entire functions. The convergence behavior for more general kernels was analyzed in [22].

Further extensions and generalizations of the sampling theorem are treated in the overview article [14], which contains numerous references itself, and in $[16,17]$. Recent developments in sampling theory can be found in $[11,12,18]$.

Often, the interest is not in the signal itself but in some processed version of it. This might be the derivative, the Hilbert transform, or the result of any other 
linear operator $T$. Thus, the goal is to approximate the desired transformation $T f$ of a signal $f$ by an approximation process, which uses only finitely many samples of the signal, taken at or above Nyquist rate. Exactly as in the case of signal reconstruction, the convergence behavior is important for practical applications.

One possible application for this sampling-based signal processing approach is in sensor networks. There, the signal of interest is some physical quantity, for example, temperature or electric field intensity, and the task is to approximate some transformation of this signal by using only the samples of the signal, which are produced by the sensors.

\section{Comparison: Analog and Digital Signal Processing}

In order to continue, we need some notation and definitions. Let $\hat{f}$ denote the Fourier transform of a function $f$, where $\hat{f}$ is to be understood in the distributional sense. $L^{p}(\mathbb{R}), 1 \leq p<\infty$, is the space of all the $p$ th power Lebesgue integrable functions on $\mathbb{R}$, with the usual norm $\|\cdot\|_{p}$, and $L^{\infty}(\mathbb{R})$ the space of all functions for which the essential supremum norm $\|\cdot\|_{\infty}$ is finite. $C_{0}^{\infty}\left[t_{1}, t_{2}\right]$ denotes the space of all infinitely differentiable functions on $\mathbb{R}$ whose support is in $\left[t_{1}, t_{2}\right]$. Furthermore, $l^{p}, 1 \leq p<\infty$, is the space of all sequences such that the $p$-norm $\|\cdot\|_{p}$ is finite, and $l^{\infty}$ denotes the space of bounded sequences with the supremum norm $\|\cdot\|_{l^{\infty}}$.

For $\sigma>0$, let $\mathcal{B}_{\sigma}$ be the set of all entire functions $f$ with the property that for all $\epsilon>0$ there exists a constant $C(\epsilon)$ with $|f(z)| \leq C(\epsilon) \exp ((\sigma+\epsilon)|z|)$ for all $z \in \mathbb{C}$. The Bernstein space $\mathcal{B}_{\sigma}^{p}$ consists of all signals in $\mathcal{B}_{\sigma}$, whose restriction to the real line is in $L^{p}(\mathbb{R}), 1 \leq p \leq \infty$. A signal in $\mathcal{B}_{\sigma}^{p}$ is called bandlimited to $\sigma$. By the Paley-Wiener-Schwartz theorem, the Fourier transform of a signal bandlimited to $\sigma$ is supported in $[-\sigma, \sigma]$. For $1 \leq p \leq 2$ the Fourier transform is defined in the classical and for $p>2$ in the distributional sense. It is well known that $\mathcal{B}_{\sigma}^{p} \subset \mathcal{B}_{\sigma}^{s}$ for $1 \leq p \leq s \leq \infty$. Hence, every signal $f \in \mathcal{B}_{\sigma}^{p}, 1 \leq p \leq \infty$, is bounded.

For $\sigma>0$ and $1 \leq p \leq \infty$, we denote by $\mathcal{P} \mathcal{W}_{\sigma}^{p}$ the Paley-Wiener space of signals $f$ with a representation $f(z)=\frac{1}{2 \pi} \int_{-\sigma}^{\sigma} g(\omega) \mathrm{e}^{i z \omega} \mathrm{d} \omega, z \in \mathbb{C}$, for some $g \in L^{p}[-\sigma, \sigma]$. If $f \in \mathcal{P} \mathcal{W}_{\sigma}^{p}$ then $g(\omega)=\hat{f}(\omega)$. The norm for $\mathcal{P} \mathcal{W}_{\sigma}^{p}, 1 \leq p<\infty$, is given by $\|f\|_{\mathcal{P} \mathcal{W}_{\sigma}^{p}}=\left(\frac{1}{2 \pi} \int_{-\sigma}^{\sigma}|\hat{f}(\omega)|^{p} \mathrm{~d} \omega\right)^{1 / p}$.

As a consequence of Parseval's equality we have $\mathcal{B}_{\pi}^{2}=\mathcal{P} \mathcal{W}_{\pi}^{2}$. Furthermore, the Hausdorff-Young inequality leads to $\mathcal{B}_{\pi}^{q} \supset \mathcal{P W}_{\pi}^{p}$ for $1<p \leq 2,1 / p+1 / q=1$, and Hölder's inequality to $\mathcal{P} \mathcal{W}_{\sigma}^{p} \supset \mathcal{P} \mathcal{W}_{\sigma}^{s}$ for $1 \leq p<s \leq \infty$. Moreover, it holds that $\|f\|_{\infty} \leq\|f\|_{\mathcal{P} \mathcal{W}^{1}}$.

Since it is desirable to have a stable reconstruction for as large a space of signals as possible, we focus our analysis on the space $\mathcal{P} \mathcal{W}_{\pi}^{1}$, because $\mathcal{P} \mathcal{W}_{\pi}^{1}$ is the largest space in the scale of Paley-Wiener spaces with bandwidth $\pi$. 
Next, we discuss some basic properties of bandlimited signals that illustrate the relationship between continuous-time and discrete-time signals and, thus, the connection between analog and digital signal processing. The spaces $\mathcal{B}_{\pi}^{p}$ and $l^{p}$ for $1<p<\infty$, for instance, can be used interchangeably because of the isomorphism between them. This isomorphism is established by the linear sampling operator $S_{\pi}: \mathcal{B}_{\pi}^{p} \rightarrow l^{p}, f \mapsto(f(k))_{k \in \mathbb{Z}}$ and the linear interpolation operator $T_{\pi}: l^{p} \rightarrow \mathcal{B}_{\pi}^{p},(f(k))_{k \in \mathbb{Z}} \mapsto \sum_{k=-\infty}^{\infty} f(k) \frac{\sin (\pi(t-k))}{\pi(t-k)}$, which is given by the Shannon sampling series.

A very useful tool in the convergence analysis of the Shannon sampling series is the Plancherel-Pólya theorem [27, 20].

Plancherel-Pólya Theorem. Let $1<p<\infty$. There are two constants $C_{1}(p)>0$ and $C_{2}(p)>0$, depending only on $p$, such that for all $f \in \mathcal{B}_{\pi}^{p}$

$$
C_{1}(p)\left(\sum_{k=-\infty}^{\infty}|f(k)|^{p}\right)^{1 / p} \leq\left(\int_{-\infty}^{\infty}|f(t)|^{p} \mathrm{~d} t\right)^{1 / p} \leq C_{2}(p)\left(\sum_{k=-\infty}^{\infty}|f(k)|^{p}\right)^{1 / p} .
$$

Note, the first inequality of (1) also holds for $p=1$ and $p=\infty$. It is known in mathematical literature that the second inequality (1) cannot be valid for $p=1$ and $p=\infty$ (see corresponding remarks in [27, p. 11 and p. 22]).

For signals $f \in \mathcal{B}_{\pi}^{p}, 1<p<\infty$, the convergence of the Shannon sampling series can be shown easily. Since

$$
\left(S_{N} f\right)(t):=\sum_{k=-N}^{N} f(k) \frac{\sin (\pi(t-k))}{\pi(t-k)}
$$

is a finite linear combination of sinc functions, for $f \in \mathcal{B}_{\pi}^{p}, 1<p<\infty, f-S_{N} f$ is in $\mathcal{B}_{\pi}^{p}$, too. Therefore,

$$
\left\|f-S_{N} f\right\|_{p} \leq C_{2}(p)\left(\sum_{k=-\infty}^{\infty}\left|f(k)-\left(S_{N} f\right)(k)\right|^{p}\right)^{1 / p} \leq C_{2}(p)\left(\sum_{|k|>N}|f(k)|^{p}\right)^{1 / p},
$$

and consequently $\lim _{N \rightarrow \infty}\left\|f-S_{N} f\right\|_{p}=0$. Note that $\left\|f-S_{N} f\right\|_{\infty} \leq C_{3}(p) \| f-$ $S_{N} f \|_{p}$, for some constant $C_{3}(p)$. Thus, for $f \in \mathcal{B}_{\pi}^{p}, 1<p<\infty$, the peak error in the approximation $\sup _{t \in \mathbb{R}}\left|f(t)-S_{N} f(t)\right|$, made by the truncation of the Shannon sampling series to $N$ summands, can be bounded above and goes to zero for $N \rightarrow \infty$. Since $\mathcal{B}_{\pi}^{1} \subset \mathcal{B}_{\pi}^{2}$, this result is also valid for $p=1$.

Furthermore, we have the uniform convergence of the Shannon sampling series for the for the Paley-Wiener spaces $\mathcal{P} \mathcal{W}_{\pi}^{p}, p>1$, because $\mathcal{B}_{\pi}^{q} \supset \mathcal{P} \mathcal{W}_{\pi}^{p}$ for $1<p \leq 2,1 / p+1 / q=1$, and $\mathcal{P} \mathcal{W}_{\sigma}^{p} \subset \mathcal{P} \mathcal{W}_{\sigma}^{2}$ for $p>2$.

For $f \in \mathcal{P W}_{\pi}^{1}$ the situation is different. In [2] it has been shown for a very general class of axiomatically defined reconstruction processes that a stable reconstruction is not possible in general. 


\subsection{Sampling-based Signal Processing}

In this paper we analyze the question how signal processing operations, defined for continuous-time signals, can be approximated by using only the samples of the signal. In order to do this we need a reconstruction process, which generates the continuous time-signal out of the samples. We approach this problem not by considering one specific reconstruction process, e.g., the Shannon sampling series, but by doing the investigation for a whole class of reconstruction processes. The analyzed reconstruction processes $A_{N, \phi}^{a}, N \in \mathbb{N}$, are of the general structure

$$
\left(A_{N, \phi}^{a} f\right)(t):=\sum_{k=-N}^{N} f\left(\frac{k}{a}\right) \phi\left(t-\frac{k}{a}\right),
$$

where $a \geq 1$ and $\phi \in \mathcal{B}_{a \pi}^{\infty}$ is such that $A_{N, \phi}^{a}$ has the M-property.

Definition 1. For given $a \geq 1$ and $\phi \in \mathcal{B}_{a \pi}^{\infty}$, we say a reconstruction process $A_{N, \phi}^{a}$ has the M-property if

$$
\lim _{N \rightarrow \infty}\left\|f-A_{N, \phi}^{a} f\right\|_{\mathcal{P} \mathcal{W}_{a \pi}^{2}}=0
$$

for all $f \in \mathcal{P} \mathcal{W}_{\pi}^{2}$.

The M-property, where $\mathrm{M}$ stands for minimum requirement, is not a practical restriction. It is a kind of minimum requirement, i.e., we require at least a reconstruction for signals in $\mathcal{P} \mathcal{W}_{\pi}^{2}$.

Example 1. If $a>1$, many kernels $\phi \in \mathcal{B}_{a \pi}^{\infty}$ are possible. For example, kernels $\phi$ with trapezoidal shape in the frequency domain, i.e.,

$$
\hat{\phi}(\omega)= \begin{cases}\frac{1}{a} & |\omega| \leq \pi \\ \frac{|\omega|-a \pi}{a \pi(1-a)} & \pi<|\omega|<a \pi \\ 0 & |\omega| \geq a \pi\end{cases}
$$

or kernels $\phi$ with a cosine roll-off characteristic in the frequency domain, i.e.,

$$
\hat{\phi}(\omega)= \begin{cases}\frac{1}{a} & |\omega| \leq \pi \\ \frac{1}{2 a}\left(1-\sin \left[\frac{\pi}{a-1}\left(\left|\frac{\omega}{\pi}\right|-\frac{a+1}{2}\right)\right]\right) & \pi<|\omega|<a \pi \\ 0 & |\omega| \geq a \pi\end{cases}
$$

can be used. If $a=1$, the only possible kernel is the sinc kernel

$$
\phi(t)=\frac{\sin (\pi t)}{\pi t} .
$$

In Section 3 we will see that all kernels above belong to the class $\mathcal{M}(a)$, and that all kernels in $\mathcal{M}(a)$ lead to reconstruction processes that have the M-property. 
Observation 1. Since for all $a \geq 1$ and each finite $N \in \mathbb{N}$ there is a $f_{N} \in$ $\mathcal{P W}_{\pi}^{2}$ such that $f_{N}(0)=1$ and $f_{N}(k / a)=0,1 \leq|k| \leq N$, it follows for all reconstruction processes that have the M-property that $\phi \in \mathcal{P} \mathcal{W}_{a \pi}^{2}$.

Definition 2. $\mathcal{W}$ is the space of continuous signals $f$ with the property that $\hat{f} \in L^{1}(\mathbb{R})$ exists in the distributional sense and $f(t)=\frac{1}{2 \pi} \int_{-\infty}^{\infty} \hat{f}(\omega) e^{i \omega t} \mathrm{~d} \omega$. The norm is given by $\|f\|_{\mathcal{W}}=\frac{1}{2 \pi}\|\hat{f}\|_{1}$.

Definition 3. By $\mathcal{L}$ we denote the set of translation invariant, linear, and bounded operators $T: \mathcal{W} \rightarrow \mathcal{W}$, and by $\mathcal{L}\left(\mathcal{P} \mathcal{W}_{\pi}^{p}\right), 1 \leq p \leq \infty$, the set of translation invariant, linear, and bounded operators $T: \mathcal{P} \mathcal{W}_{\pi}^{p} \rightarrow \mathcal{P} \mathcal{W}_{\pi}^{p}$.

Observation 2. For every $T \in \mathcal{L}$, there is exactly one function $\hat{h}_{T} \in L^{\infty}(\mathbb{R})$ such that

$$
(T f)(t)=\frac{1}{2 \pi} \int_{-\infty}^{\infty} \hat{h}_{T}(\omega) \hat{f}(\omega) \mathrm{e}^{i \omega t} \mathrm{~d} \omega
$$

for all $f \in \mathcal{W}$, and the operator norm $\|T\|_{\mathcal{L}}:=\sup _{\|f\|_{\mathcal{W} \leq 1}}\|T f\|_{\mathcal{W}}$ is given by $\|T\|_{\mathcal{L}}=\left\|\hat{h}_{T}\right\|_{\infty}$.

Observation 2 allows us to identify the systems in $\mathcal{L}$ with functions in $L^{\infty}(\mathbb{R})$ and gives a representation in the form of the integral (3), which is valid for all systems in $\mathcal{L}$. For the sake of completeness, the proof of Observation 2 is given in Appendix A. Conversely, every function $\hat{h}_{T} \in L^{\infty}(\mathbb{R})$ defines an operator in $\mathcal{L}$. Thus, the space $\mathcal{L}$ is isometric isomorph to $L^{\infty}(\mathbb{R})$.

Moreover, a look at the proof of Observation 2 reveals that every translation invariant, linear, and bounded operator $T: \mathcal{W} \rightarrow L^{\infty}(\mathbb{R})$ has the same representation (3) with a unique function $\hat{h}_{T} \in L^{\infty}(\mathbb{R})$, which implies that $T \in \mathcal{L}$ and in particular, that the range of $T$ is $\mathcal{W}$. Furthermore, since $\|f\|_{\infty} \leq\|f\|_{\mathcal{W}}$, every operator $T \in \mathcal{W}$ is a translation invariant, linear, and bounded operator $T: \mathcal{W} \rightarrow L^{\infty}(\mathbb{R})$. This behavior is specific to the space $\mathcal{W}$ and does not necessarily hold for other spaces. For example, the range of a translation invariant, linear and bounded operator $T: L^{2}(\mathbb{R}) \rightarrow L^{\infty}(\mathbb{R})$ is not $L^{2}(\mathbb{R})$ in general.

Furthermore, it can be shown that every $T \in \mathcal{L}\left(\mathcal{P} \mathcal{W}_{\pi}^{p}\right), 1 \leq p \leq 2$, has the representation

$$
(T f)(t)=\frac{1}{2 \pi} \int_{-\pi}^{\pi} \hat{h}_{T}(\omega) \hat{f}(\omega) \mathrm{e}^{i \omega t} \mathrm{~d} \omega
$$

with $\hat{h}_{T} \in L^{\infty}[-\pi, \pi]$, and that the operator norm is given by $\|T\|_{\mathcal{L}\left(\mathcal{P} \mathcal{W}_{\pi}^{p}\right)}=$ $\left\|\hat{h}_{T}\right\|_{\infty}$. Consequently, we do not need to distinguish between operators in $\mathcal{L}\left(\mathcal{P} \mathcal{W}_{\pi}^{1}\right)$ and, in $\mathcal{L}\left(\mathcal{P} \mathcal{W}_{\pi}^{2}\right)$, because both can be identified with $\hat{h} \in L^{\infty}[-\pi, \pi]$.

Example 2. The Hilbert transform $H$ is an example for an operator in $\mathcal{L}$. The Hilbert transform $\tilde{f}$ of a signal $f \in \mathcal{W}$ is defined by

$$
\tilde{f}(t)=(H f)(t)=\frac{1}{2 \pi} \int_{-\infty}^{\infty}(-i \operatorname{sgn}(\omega)) \hat{f}(\omega) \mathrm{e}^{i \omega t} \mathrm{~d} \omega
$$


where sgn denotes the signum function. Obviously, $\|H\|_{\mathcal{L}}=1$ and consequently $H \in \mathcal{L}$.

Example 3. An example for an operator in $\mathcal{L}\left(\mathcal{P} \mathcal{W}_{\pi}^{1}\right)$ is the differential operator $D$, which is defined by

$$
(D f)(t)=\frac{1}{2 \pi} \int_{-\pi}^{\pi} i \omega \hat{f}(\omega) \mathrm{e}^{i \omega t} \mathrm{~d} \omega .
$$

Remark 1. All preceding considerations have been made for the bandwidth $\pi$. However, they can be easily adapted to general bandwidths $\sigma>0$.

Of course, it is natural to look for a way to calculate $T f$ from the samples of $f$. This is the key idea of sampling-based signal processing: the whole signal is not used to calculate some transformation of the signal, but rather the samples of the signal. Obviously, this corresponds to the natural situation in digital signal processing, where only samples of the signal are available. Thus, the question whether and how $T f$ can be calculated from the samples of $f$ is of practical importance. Sampling-based signal processing should be potentially possible because $f$ is, as a bandlimited signal, uniquely determined by its samples, i.e., the whole information about the signal is contained in the samples.

For $f \in \mathcal{P} \mathcal{W}_{\pi}^{2}$ the situation is simple: We have

$$
(T f)(t)=\sum_{k=-\infty}^{\infty} f(k) h_{T}(t-k)
$$

due to the convergence of the Shannon sampling series in the $\mathcal{P} \mathcal{W}_{\pi}^{2}$-norm and the continuity and linearity of $T \in \mathcal{L}\left(\mathcal{P} \mathcal{W}_{\pi}^{2}\right)$. The series in (5) converges in the $\mathcal{P} \mathcal{W}_{\pi}^{2}$-norm and consequently uniformly on $\mathbb{R}$. Because of the convergence behavior of the Shannon sampling series for $f \in \mathcal{P} \mathcal{W}_{\pi}^{1}$, in particular because the Shannon sampling series is not convergent in the $\mathcal{P} \mathcal{W}_{\pi}^{1}$-norm for signals in $\mathcal{P} \mathcal{W}_{\pi}^{1}$ in general [1], we cannot expect such a behavior for $\mathcal{P} \mathcal{W}_{\pi}^{1}$.

By considering oversampling and a variety of kernels, we analyze the convergence behavior for a whole class of approximation processes, not only the Shannon sampling series. For $T \in \mathcal{L}$ we have

$$
\left(T_{N, \phi}^{a} f\right)(t):=\left(T A_{N, \phi}^{a} f\right)(t)=\sum_{k=-N}^{N} f\left(\frac{k}{a}\right)(T \phi)\left(t-\frac{k}{a}\right)
$$

by applying $T$ on (2). The expression $T \phi$, and thus equation (6), is well defined, because $\phi \in \mathcal{P} \mathcal{W}_{a \pi}^{2} \subset \mathcal{P} \mathcal{W}_{a \pi}^{1} \subset \mathcal{W}$ by Observation 1 . Note that if the samples $\{f(k / a)\}_{k=-N}^{N}$ are known, then (6) is a linear method to calculate an approximation of $T f$. For $N \rightarrow \infty$ one could hope that $T_{N, \phi}^{a} f$ is close to $T f$ in some suitable norm. 
In many situations, oversampling eliminates convergence problems, because it creates an additional degree of freedom for the kernel choice (see Example 1). In particular, it is possible to use kernels that have a better concentration in the time domain and thus lead to a better convergence behavior of the sampling series. However, as we show in Section 4, oversampling cannot improve the convergence behavior of (6) in general, i.e., there are operators $T \in \mathcal{L}$ for which oversampling is useless.

\section{Characterization of Sampling Series Kernels for $\mathcal{P} \mathcal{W}_{\pi}^{2}$}

In Section 2 we have introduced and motivated sampling-based signal processing. Next, general reconstruction processes shall be analyzed. We start by characterizing the kernels $\phi$ for which the reconstruction process

$$
\left(A_{N, \phi}^{a} f\right)(t)=\sum_{k=-N}^{N} f\left(\frac{k}{a}\right) \phi\left(t-\frac{k}{a}\right),
$$

$a \geq 1$, fulfills the M-property, i.e., it converges to $f$ in the $\mathcal{P} \mathcal{W}_{\pi}^{2}$-norm for all $f \in \mathcal{P} \mathcal{W}_{\pi}^{2}$.

Definition 4. $\mathcal{M}(a), a>1$, is the set of functions $\phi \in \mathcal{B}_{a \pi}^{1}$ with $\hat{\phi}(\omega)=1 / a$ for $|\omega| \leq \pi$.

The functions in $\mathcal{M}(a), a>1$, are suitable kernels for the sampling series (7) because for all $f \in \mathcal{P} \mathcal{W}_{\pi}^{1}$ and $a>1$ we have $\lim _{N \rightarrow \infty}\left\|f-A_{N, \phi}^{a} f\right\|_{\infty}=0$ if $\phi \in \mathcal{M}(a)$. We do not prove this statement here because it follows from Corollary 2 and Lemma 1. Furthermore, for all $\phi \in \mathcal{M}(a), a>1$, and all $f \in \mathcal{P} \mathcal{W}_{\pi}^{2}$ we have $\lim _{N \rightarrow \infty}\left\|f-A_{N, \phi}^{a} f\right\|_{\mathcal{P} \mathcal{W}_{a \pi}^{2}}=0[8]$.

Definition 5. $\mathcal{M}^{2}(a), a \geq 1$, denotes the set of all $\phi \in \mathcal{P} \mathcal{W}_{a \pi}^{2}$ such that $\lim _{N \rightarrow \infty}\left\|f-A_{N, \phi}^{a} f\right\|_{\mathcal{P} \mathcal{W}_{a \pi}^{2}}=0$ for all $f \in \mathcal{P} \mathcal{W}_{\pi}^{2}$.

Remark 2. The set $\mathcal{M}^{2}(1)$ contains only the sinc kernel $\sin (\pi t) /(\pi t)$. Furthermore, by Observation $1, \phi \in \mathcal{M}^{2}(a), a \geq 1$, if and only if $\phi \in \mathcal{B}_{a \pi}^{\infty}$ and $A_{N, \phi}^{a}$ fulfills the M-property.

Obviously, the sets $\mathcal{M}(a)$ and $\mathcal{M}^{2}(a)$ are related to each other by the inclusion $\mathcal{M}(a) \subset \mathcal{M}^{2}(a), a \geq 1$. This relation can be more precisely characterized by the following two lemmas, Lemma 1 and Lemma 2 .

Lemma 1. Let $\phi \in \mathcal{B}_{a \pi}^{1}$. Then there exists a constant $C_{4}$ such that $\sum_{k=-\infty}^{\infty} \mid \phi(t-$ $k / a) \mid \leq C_{4}$ for all $t \in \mathbb{R}$. 
Proof. For $g$ with $\hat{g} \in C_{0}^{\infty}[-(a+1) \pi,(a+1) \pi]$ and $\hat{g}(\omega)=1,|\omega| \leq a \pi$, we have $\phi(t)=\int_{-\infty}^{\infty} \phi(\tau) g(t-\tau) \mathrm{d} \tau$ and

$$
\sum_{k=-N}^{N}\left|\phi\left(t-\frac{k}{a}\right)\right| \leq \int_{-\infty}^{\infty}|\phi(\tau)| \sum_{k=-N}^{N}\left|g\left(t-\frac{k}{a}-\tau\right)\right| \mathrm{d} \tau
$$

Since $\sum_{k=-N}^{N}|g(t-k / a-\tau)| \leq C_{5}$, we obtain $\sum_{k=-\infty}^{\infty}|\phi(t-k / a)| \leq C_{5}\|\phi\|_{\mathcal{B}_{a \pi}^{1}}$, which completes the proof.

Lemma 2. $\phi \in \mathcal{M}(a)$ if and only if $\phi \in \mathcal{M}^{2}(a)$ and there exists a positive constant $C_{4}$ such that $\sum_{k=-\infty}^{\infty}|\phi(t-k / a)| \leq C_{4}$ for all $t \in \mathbb{R}$.

Proof. " $\Rightarrow$ ": Since, $\mathcal{M}(a) \subset \mathcal{M}^{2}(a), a \geq 1$, it remains to show that there exists a positive constant $C_{4}$ such that $\sum_{k=-\infty}^{\infty}|\phi(t-k / a)| \leq C_{4}$ for all $t \in \mathbb{R}$. However, this is exactly the statement of Lemma 1.

" $\Leftarrow$ ": By assumption $a \int_{0}^{1 / a} \sum_{k=-\infty}^{\infty}|\phi(t-k / a)| \mathrm{d} t \leq C_{4}$, and since

$$
a \int_{0}^{1 / a} \sum_{k=-\infty}^{\infty}\left|\phi\left(t-\frac{k}{a}\right)\right| \mathrm{d} t=a \sum_{k=-\infty}^{\infty} \int_{-k / a}^{(1-k) / a}|\phi(t)| \mathrm{d} t=a \int_{-\infty}^{\infty}|\phi(t)| \mathrm{d} t
$$

by the monotone convergence theorem, we have $a\|\phi\|_{\mathcal{B}_{a \pi}^{1}} \leq C_{4}$.

Remark 3. Lemma 2 shows that the class of kernels $\mathcal{M}^{2}(a)$ is very large. This is important in order not to impose too large a restriction on the permissible kernels.

The set $\mathcal{M}^{2}(a)$ can be explicitly characterized on the basis of Theorem 1 , in which the functional $C^{2}: \mathcal{P} \mathcal{W}_{\pi}^{2} \rightarrow \mathbb{R}$,

$$
C^{2}(\phi)=\sup _{N \in \mathbb{N}}\left(\sup _{\|f\|_{\mathcal{P} \mathcal{W}_{\pi}^{2}} \leq 1}\left(\frac{1}{2 \pi} \int_{\pi \leq|\omega| \leq a \pi}\left|a\left(F_{N}^{a} \hat{f}\right)(\omega)\right|^{2}|\hat{\phi}(\omega)|^{2} \mathrm{~d} \omega\right)^{\frac{1}{2}}\right),
$$

with

$$
\left(F_{N}^{a} \hat{f}\right)(\omega)=\frac{1}{a} \sum_{k=-N}^{N} f\left(\frac{k}{a}\right) \mathrm{e}^{-i \omega k / a}
$$

plays an important role.

Theorem 1. Let $a \geq 1$. Then $\phi \in \mathcal{M}^{2}(a)$ if and only if $\phi \in \mathcal{P} \mathcal{W}_{a \pi}^{2}$ and

1. $\hat{\phi}(\omega)=1 / a$ a.e. for $|\omega| \leq \pi$; and

2. $C^{2}(\phi)<\infty$. 
Remark 4. The conditions 1 and 2 of Theorem 1 have a descriptive interpretation. Condition 1 basically says that $\hat{\phi}$ should be constant in the passband, and $C^{2}(\phi)$ in condition 2 describes the weighted out-of-band energy of $\phi$.

Proof. First part, " $\Rightarrow$ ": Let $a \geq 1$ and $\phi \in \mathcal{M}^{2}(a)$ be arbitrary but fixed. For each $N \in \mathbb{N}$ we have

$$
\left\|A_{N, \phi}^{a} f\right\|_{\mathcal{P} \mathcal{W}_{a \pi}^{2}} \leq\|f\|_{\infty} \sum_{k=-N}^{N}\|\phi\|_{\mathcal{P} \mathcal{W}_{\pi}^{2}} \leq\|f\|_{\mathcal{P} \mathcal{W}_{\pi}^{2}}(2 N+1)\|\phi\|_{\mathcal{P} \mathcal{W}_{\pi}^{2}},
$$

which shows that $A_{N, \phi}^{a}: \mathcal{P} \mathcal{W}_{\pi}^{2} \rightarrow \mathcal{P} \mathcal{W}_{\pi}^{2}$ is a bounded linear operator. Moreover, since $\phi \in \mathcal{M}^{2}(a)$, we have, according to Definition $5, \lim _{N \rightarrow \infty}\left\|A_{N, \phi}^{a} f\right\|_{\mathcal{P} \mathcal{W}_{a \pi}^{2}}=$ $\|f\|_{\mathcal{P} \mathcal{W}_{a \pi}^{2}}$, and consequently $\sup _{N \in \mathbb{N}}\left\|A_{N, \phi}^{a} f\right\|_{\mathcal{P} \mathcal{W}_{a \pi}^{2}}<\infty$ for all $f \in \mathcal{P} \mathcal{W}_{\pi}^{2}$. Thus, it follows by the Banach-Steinhaus theorem [24, p. 98] that there exists a constant $C_{6}$ such that $\left\|A_{N, \phi}^{a} f\right\|_{\mathcal{P} \mathcal{W}_{a \pi}^{2}} \leq C_{6}\|f\|_{\mathcal{P} \mathcal{W}_{\pi}^{2}}$ for all $f \in \mathcal{P} \mathcal{W}_{\pi}^{2}$ and all $N \in \mathbb{N}$. Since

$$
\begin{aligned}
& \left\|A_{N, \phi}^{a} f\right\|_{\mathcal{P} \mathcal{W}_{a \pi}^{2}}^{2}=\frac{1}{2 \pi} \int_{-a \pi}^{a \pi}\left|\sum_{k=-N}^{N} f\left(\frac{k}{a}\right) \hat{\phi}(\omega) \mathrm{e}^{-i \omega k / a}\right|^{2} \mathrm{~d} \omega \\
& =\frac{1}{2 \pi} \int_{-\pi}^{\pi}\left|a\left(F_{N}^{a} \hat{f}\right)(\omega)\right|^{2}|\hat{\phi}(\omega)|^{2} \mathrm{~d} \omega+\frac{1}{2 \pi} \int_{\pi \leq|\omega| \leq a \pi}\left|a\left(F_{N}^{a} \hat{f}\right)(\omega)\right|^{2}|\hat{\phi}(\omega)|^{2} \mathrm{~d} \omega \\
& \geq \frac{1}{2 \pi} \int_{-\pi}^{\pi}\left|a\left(F_{N}^{a} \hat{f}\right)(\omega)\right|^{2}|\hat{\phi}(\omega)|^{2} \mathrm{~d} \omega,
\end{aligned}
$$

we obtain

$$
\left(\frac{1}{2 \pi} \int_{-\pi}^{\pi}\left|a\left(F_{N}^{a} \hat{f}\right)(\omega)\right|^{2}|\hat{\phi}(\omega)|^{2} \mathrm{~d} \omega\right)^{\frac{1}{2}} \leq C_{6}\|f\|_{\mathcal{P} \mathcal{W}_{\pi}^{2}}
$$

for all $f \in \mathcal{P} \mathcal{W}_{\pi}^{2}$ and all $N \in \mathbb{N}$.

Furthermore, for all $f \in \mathcal{P} \mathcal{W}_{\pi}^{2}$ with $\|f\|_{\mathcal{P W}_{\pi}^{2}} \leq 1$ and $\hat{f} \in C_{0}^{\infty}[-\pi, \pi]$, we have $\lim _{N \rightarrow \infty} \max _{\omega \in[-\pi, \pi]}\left|\hat{f}(\omega)-\left(F_{N}^{a} \hat{f}\right)(\omega)\right|=0\left[31\right.$, p. 57] and since $\phi \in \mathcal{P} \mathcal{W}_{a \pi}^{2}$, it follows

$$
\begin{aligned}
& \left(\frac{1}{2 \pi} \int_{-\pi}^{\pi}|\hat{f}(\omega)|^{2}|\hat{\phi}(\omega)|^{2} \mathrm{~d} \omega\right)^{\frac{1}{2}} \\
& =\left(\frac{1}{2 \pi} \int_{-\pi}^{\pi}\left|\hat{f}(\omega)-\left(F_{N}^{a} \hat{f}\right)(\omega)+\left(F_{N}^{a} \hat{f}\right)(\omega)\right|^{2}|\hat{\phi}(\omega)|^{2} \mathrm{~d} \omega\right)^{\frac{1}{2}} \\
& \leq\left(\frac{1}{2 \pi} \int_{-\pi}^{\pi}\left|\hat{f}(\omega)-\left(F_{N}^{a} \hat{f}\right)(\omega)\right|^{2}|\hat{\phi}(\omega)|^{2} \mathrm{~d} \omega\right)^{\frac{1}{2}}+\left(\frac{1}{2 \pi} \int_{-\pi}^{\pi}\left|\left(F_{N}^{a} \hat{f}\right)(\omega)\right|^{2}|\hat{\phi}(\omega)|^{2} \mathrm{~d} \omega\right)^{\frac{1}{2}} \\
& \leq\left(\max _{\omega \in[-\pi, \pi]}\left|\hat{f}(\omega)-\left(F_{N}^{a} \hat{f}\right)(\omega)\right|^{2}\right)^{\frac{1}{2}}\|\phi\|_{\mathcal{P} \mathcal{W}_{\pi}^{2}}+\frac{1}{a^{2}} C_{6}\|f\|_{\mathcal{P} \mathcal{W}_{\pi}^{2}},
\end{aligned}
$$


i.e.,

$$
\frac{1}{2 \pi} \int_{-\pi}^{\pi}|\hat{f}(\omega)|^{2}|\hat{\phi}(\omega)|^{2} \mathrm{~d} \omega \leq C_{7}
$$

Hence, $\operatorname{ess}_{\sup _{\omega \in[-\pi, \pi]}}|\hat{\phi}(\omega)|<\infty$, i.e, $\hat{\phi}$ restricted to $[-\pi, \pi]$ is in $L^{\infty}$. Moreover,

$$
\begin{aligned}
& \left(\frac{1}{2 \pi} \int_{-\pi}^{\pi}|\hat{f}(\omega)|^{2}|1-a \hat{\phi}(\omega)|^{2} \mathrm{~d} \omega\right)^{\frac{1}{2}} \\
& =\left(\frac{1}{2 \pi} \int_{-\pi}^{\pi}\left|\hat{f}(\omega)-a\left(F_{N}^{a} \hat{f}\right)(\omega) \hat{\phi}(\omega)+a\left(F_{N}^{a} \hat{f}\right)(\omega) \hat{\phi}(\omega)-a \hat{f}(\omega) \hat{\phi}(\omega)\right|^{2} \mathrm{~d} \omega\right)^{\frac{1}{2}} \\
& \leq\left(\frac{1}{2 \pi} \int_{-\pi}^{\pi}\left|\hat{f}(\omega)-a\left(F_{N}^{a} \hat{f}\right)(\omega) \hat{\phi}(\omega)\right|^{2} \mathrm{~d} \omega\right)^{\frac{1}{2}} \\
& \quad+\left(\frac{1}{2 \pi} \int_{-\pi}^{\pi} a^{2}\left|\left(F_{N}^{a} \hat{f}\right)(\omega)-\hat{f}(\omega)\right|^{2}|\hat{\phi}(\omega)|^{2} \mathrm{~d} \omega\right)^{\frac{1}{2}} \\
& \leq\left(\frac{1}{2 \pi} \int_{-\pi}^{\pi}\left|\hat{f}(\omega)-a\left(F_{N}^{a} \hat{f}\right)(\omega) \hat{\phi}(\omega)\right|^{2} \mathrm{~d} \omega\right)^{\frac{1}{2}} \\
& \quad+\operatorname{esssup}_{\omega \in[-\pi, \pi]}|\hat{\phi}(\omega)|\left(\frac{1}{2 \pi} \int_{-\pi}^{\pi} a^{2}\left|\left(F_{N}^{a} \hat{f}\right)(\omega)-\hat{f}(\omega)\right|^{2} \mathrm{~d} \omega\right)^{\frac{1}{2}}
\end{aligned}
$$

Since $\lim _{N \rightarrow \infty}\left\|f-A_{N, \phi}^{a} f\right\|_{\mathcal{P} \mathcal{W}_{a \pi}^{2}}=0$ and

$$
\left\|f-A_{N, \phi}^{a} f\right\|_{\mathcal{P} \mathcal{W}_{a \pi}^{2}}^{2} \geq \frac{1}{2 \pi} \int_{-\pi}^{\pi}\left|\hat{f}(\omega)-a\left(F_{N}^{a} \hat{f}\right)(\omega) \hat{\phi}(\omega)\right|^{2} \mathrm{~d} \omega
$$

by the same steps as in (8), it follows that

$$
\lim _{N \rightarrow \infty} \frac{1}{2 \pi} \int_{-\pi}^{\pi}\left|\hat{f}(\omega)-a\left(F_{N}^{a} \hat{f}\right)(\omega) \hat{\phi}(\omega)\right|^{2} \mathrm{~d} \omega=0
$$

for all $f \in \mathcal{P} \mathcal{W}_{\pi}^{2}$. Consequently, for $N \rightarrow \infty$ the right-hand side of (9) tends to zero and we obtain

$$
\frac{1}{2 \pi} \int_{-\pi}^{\pi}|\hat{f}(\omega)|^{2}|1-a \hat{\phi}(\omega)|^{2} \mathrm{~d} \omega=0
$$

for all $f \in \mathcal{P} \mathcal{W}_{\pi}^{2}$. Hence, $\hat{\phi}(\omega)=1 / a$ a.e. for $|\omega| \leq \pi$.

Obviously, by the same calculation as in (8),

$$
\left\|A_{N, \phi}^{a} f\right\|_{\mathcal{P} \mathcal{W}_{a \pi}^{2}}^{2} \geq \frac{1}{2 \pi} \int_{\pi \leq|\omega| \leq a \pi}\left|a\left(F_{N}^{a} \hat{f}\right)(\omega)\right|^{2}|\hat{\phi}(\omega)|^{2} \mathrm{~d} \omega
$$

and since $\sup _{\|f\|_{\mathcal{P} \mathcal{W}_{\pi}^{2}} \leq 1}\left\|A_{N, \phi}^{a} f\right\|_{\mathcal{P} \mathcal{W}_{a \pi}^{2}}<C_{6}$, for all $N \in \mathbb{N}$ we have $C^{2}(\phi)<\infty$. 
Second part, " $\Leftarrow$ ": Let conditions (1) and (2) of Theorem 1 be true. For all $f \in \mathcal{P} \mathcal{W}_{\pi}^{2}$ and all $\epsilon>0$ there exists a $g \in \mathcal{P W}_{\pi}^{2}$ with $\hat{g} \in C_{0}^{\infty}[-\pi, \pi]$ such that $\|f-g\|_{\mathcal{P W}_{\pi}^{2}}<\epsilon$. Moreover,

$$
\begin{aligned}
& \left\|f-A_{N, \phi}^{a} f\right\|_{\mathcal{P} \mathcal{W}_{a \pi}^{2}}=\left\|f-g+g-A_{N, \phi}^{a} g+A_{N, \phi}^{a}(g-f)\right\|_{\mathcal{P} \mathcal{W}_{a \pi}^{2}} \\
& \leq\|f-g\|_{\mathcal{P} \mathcal{W}_{a \pi}^{2}}+\left\|g-A_{N, \phi}^{a} g\right\|_{\mathcal{P} \mathcal{W}_{a \pi}^{2}}+\left\|A_{N, \phi}^{a}(g-f)\right\|_{\mathcal{P} \mathcal{W}_{a \pi}^{2}}
\end{aligned}
$$

The first term in (10) is bounded above by $\|f-g\|_{\mathcal{P W}_{a \pi}^{2}}=\|f-g\|_{\mathcal{P W}_{\pi}^{2}}<\epsilon$.

For the second term in (10) we obtain

$$
\begin{aligned}
\left\|g-A_{N, \phi}^{a} g\right\|_{\mathcal{P} \mathcal{W}_{a \pi}^{2}}^{2}= & \frac{1}{2 \pi} \int_{-\pi}^{\pi}\left|\hat{g}(\omega)-\left(F_{N}^{a} \hat{g}\right)(\omega)\right|^{2} \mathrm{~d} \omega \\
& +\frac{1}{2 \pi} \int_{\pi \leq|\omega| \leq a \pi}\left|a\left(F_{N}^{a} \hat{g}\right)(\omega)\right|^{2}|\hat{\phi}(\omega)|^{2} \mathrm{~d} \omega .
\end{aligned}
$$

Since $g \in C_{0}^{\infty}[-\pi, \pi]$, we have $\lim _{N \rightarrow \infty} \max _{\omega \in[-\pi, \pi]}\left|\hat{g}(\omega)-\left(F_{N}^{a} \hat{g}\right)(\omega)\right|^{2}=0$ and $\lim _{N \rightarrow \infty} \max _{\pi \leq|\omega| \leq a \pi}\left|\left(F_{N}^{a} \hat{g}\right)(\omega)\right|^{2}=0$. Thus, for all $\epsilon>0$ there exists a $N_{0}=$ $N_{0}(\epsilon)$ such that

$$
\frac{1}{2 \pi} \int_{-\pi}^{\pi}\left|\hat{g}(\omega)-\left(F_{N}^{a} \hat{g}\right)(\omega)\right|^{2} \mathrm{~d} \omega<\frac{\epsilon^{2}}{2}
$$

and

$$
\frac{1}{2 \pi} \int_{\pi \leq|\omega| \leq a \pi}\left|a\left(F_{N}^{a} \hat{g}\right)(\omega)\right|^{2}|\hat{\phi}(\omega)|^{2} \mathrm{~d} \omega<\frac{\epsilon^{2}}{2},
$$

and consequently $\left\|g-A_{N, \phi}^{a} g\right\|_{\mathcal{P} \mathcal{W}_{a \pi}^{2}}^{2}<\epsilon^{2}$ for all $N \geq N_{0}$.

The third term in (10) can be further simplified according to

$$
\begin{aligned}
\left\|A_{N, \phi}^{a}(g-f)\right\|_{\mathcal{P} \mathcal{W}_{a \pi}^{2}}^{2}= & \frac{1}{2 \pi} \int_{-\pi}^{\pi}\left|\left(F_{N}^{a}(\hat{g}-\hat{f})\right)(\omega)\right|^{2} \mathrm{~d} \omega \\
& +\frac{1}{2 \pi} \int_{\pi \leq|\omega| \leq a \pi}\left|a\left(F_{N}^{a}(\hat{g}-\hat{f})\right)(\omega)\right|^{2}|\hat{\phi}(\omega)|^{2} \mathrm{~d} \omega .
\end{aligned}
$$

First of all,

$$
\begin{aligned}
& \frac{1}{2 \pi} \int_{-\pi}^{\pi}\left|\left(F_{N}^{a}(\hat{g}-\hat{f})\right)(\omega)\right|^{2} \mathrm{~d} \omega \\
& \leq \frac{1}{2 \pi} \int_{-a \pi}^{a \pi}\left|\left(F_{N}^{a}(\hat{g}-\hat{f})\right)(\omega)\right|^{2} \mathrm{~d} \omega=\frac{1}{a} \sum_{k=-N}^{N}\left|g\left(\frac{k}{a}\right)-f\left(\frac{k}{a}\right)\right|^{2} \\
& \leq \frac{1}{a} \sum_{k=-\infty}^{\infty}\left|g\left(\frac{k}{a}\right)-f\left(\frac{k}{a}\right)\right|^{2}=\|g-f\|_{\mathcal{P} \mathcal{W}_{\pi}^{2}}^{2}<\epsilon^{2}
\end{aligned}
$$


by using Parseval's theorem twice. Next, consider the operator defined by

$$
\left(R_{N, \phi}^{a} f\right)(t)=\int_{-\infty}^{\infty} \sum_{k=-N}^{N} f\left(\frac{k}{a}\right) \phi\left(t-\tau-\frac{k}{a}\right) \frac{\sin (a \pi \tau)-\sin (\pi \tau)}{\pi \tau} \mathrm{d} \tau,
$$

which is nothing but the convolution of $A_{N, \phi}^{a} f$ with the impulse response of the ideal band-pass filter with band limits $\pi$ and $a \pi$. Then it follows that

$$
\left\|R_{N, \phi}^{a} f\right\|_{\mathcal{P} \mathcal{W}_{a \pi}^{2}}=\left(\frac{1}{2 \pi} \int_{\pi \leq|\omega| \leq a \pi}\left|a\left(F_{N}^{a} \hat{f}\right)(\omega)\right|^{2}|\hat{\phi}(\omega)|^{2} \mathrm{~d} \omega\right)^{\frac{1}{2}} .
$$

Let $f \in \mathcal{P} \mathcal{W}_{\pi}^{2}$ be an arbitrary signal; then $f_{1}=f /\|f\|_{\mathcal{P} \mathcal{W}_{\pi}^{2}}$ has the norm $\left\|f_{1}\right\|_{\mathcal{P} \mathcal{W}_{\pi}^{2}}=1$.

By assumption $\left\|R_{N, \phi}^{a} f_{1}\right\|_{\mathcal{P} \mathcal{W}_{a \pi}^{2}} \leq C^{2}(\phi)$ and consequently

$$
\left\|R_{N, \phi}^{a} f\right\|_{\mathcal{P W}_{a \pi}^{2}} \leq C^{2}(\phi)\|f\|_{\mathcal{P} \mathcal{W}_{\pi}^{2}} .
$$

Thus, $\left\|A_{N, \phi}^{a}(g-f)\right\|_{\mathcal{P} \mathcal{W}_{a \pi}^{2}}^{2}<\epsilon^{2}+\left(C^{2}(\phi) \epsilon\right)^{2}$.

Inserting all partial results into (10), we obtain $\left\|f-A_{N, \phi}^{a} f\right\|_{\mathcal{P W}_{a \pi}^{2}}<\epsilon(3+$ $\left.C^{2}(\phi)\right)$ for all $N \geq N_{0}$. Since $\epsilon$ was arbitrary, the proof is complete.

\section{General Approximation Behavior}

Our analysis up to this point in the paper addresses reconstruction processes of the shape (7), which are a special case of the general approximation processes

$$
\left(T_{N, \phi}^{a} f\right)(t):=\left(T A_{N, \phi}^{a} f\right)(t)=\sum_{k=-N}^{N} f\left(\frac{k}{a}\right)(T \phi)\left(t-\frac{k}{a}\right),
$$

because (7) is obtained from (11) with $T=I d$, where $I d$ is the identity operator.

In [3] it has been shown that for every approximation process there exists an operator $T \in \mathcal{L}$ and a function $f_{1} \in \mathcal{P} \mathcal{W}_{\pi}^{1}$ such that the peak value of the approximation error $\left\|T f_{1}-T_{N, \phi}^{a} f_{1}\right\|_{\infty}, N \in \mathbb{N}$, cannot be bounded.

Theorem 2. Let $\phi \in \mathcal{M}^{2}(a), a \geq 1$. Then there exists an operator $T \in \mathcal{L}$ and signal $f_{1} \in \mathcal{P} \mathcal{W}_{\pi}^{1}$ such that

$$
\limsup _{N \rightarrow \infty}\left(\sup _{t \in \mathbb{R}}\left|\left(T f_{1}\right)(t)-\left(T_{N, \phi}^{a} f_{1}\right)(t)\right|\right)=\infty .
$$

It is even possible to state a stronger result, showing that there is a universal operator for all $\phi \in \mathcal{M}^{2}(a)$, namely the Hilbert transform, that fulfills Theorem $2[3]$. 
Consistently with (11), we introduce the abbreviation

$$
\left(H_{N, \phi}^{a} f\right)(t):=\sum_{k=-N}^{N} f\left(\frac{k}{a}\right) \tilde{\phi}\left(t-\frac{k}{a}\right)
$$

for the Hilbert transform $H$.

Theorem 3. Let $\phi \in \mathcal{M}^{2}(a), a \geq 1$. Then there exists a signal $f_{1} \in \mathcal{P} \mathcal{W}_{\pi}^{1}$ such that

$$
\limsup _{N \rightarrow \infty}\left(\sup _{t \in \mathbb{R}}\left|\left(H f_{1}\right)(t)-\left(H_{N, \phi}^{a} f_{1}\right)(t)\right|\right)=\infty .
$$

In order to prove Theorem 3 , we need Lemma 3.

Lemma 3. For all $a>1$ and $N \in \mathbb{N}$ we have

$$
\left|\sum_{k=1}^{N} \frac{\cos \left(\frac{\pi}{a} k\right)}{k}\right| \leq \frac{1}{\sin \left(\frac{\pi}{2 a}\right)} .
$$

Proof. Obviously,

$$
\left|\sum_{k=1}^{N} \frac{\cos \left(\frac{\pi}{a} k\right)}{k}\right| \leq \frac{1}{2}|\sum_{k=1}^{N} \underbrace{\mathrm{e}^{i \pi k / a}}_{=c_{k}} \underbrace{\frac{1}{k}}_{=d_{k}}|+\frac{1}{2}\left|\sum_{k=1}^{N} \mathrm{e}^{-i \pi k / a} \frac{1}{k}\right| .
$$

For the first sum on the right-hand side of (13) we have

$$
C_{k}=\sum_{l=1}^{k} c_{l}=\sum_{l=1}^{k} \mathrm{e}^{i \pi l / a}=\frac{1-\mathrm{e}^{i \pi k / a}}{1-\mathrm{e}^{i \pi / a}} \mathrm{e}^{i \pi / a}
$$

and

$$
\left|C_{k}\right| \leq \frac{1}{\sin \left(\frac{\pi}{2 a}\right)}
$$

Summation by parts gives

$$
\begin{aligned}
\left|\sum_{k=1}^{N} c_{k} d_{k}\right| & \leq\left|C_{N} d_{N}\right|+\sum_{k=1}^{N-1}\left|C_{k}\left(d_{k}-d_{k+1}\right)\right| \\
& \leq \frac{1}{\sin \left(\frac{\pi}{2 a}\right)}\left(\frac{1}{N}+\sum_{k=1}^{N-1}\left(\frac{1}{k}-\frac{1}{k+1}\right)\right) .
\end{aligned}
$$

The right-hand side of (14) can be further simplified by evaluating the telescoping series

$$
\sum_{k=1}^{N-1}\left(\frac{1}{k}-\frac{1}{k+1}\right)=1-\frac{1}{N}
$$


Thus,

$$
\left|\sum_{k=1}^{N} \mathrm{e}^{i \pi k / a} \frac{1}{k}\right| \leq \frac{1}{\sin \left(\frac{\pi}{2 a}\right)}
$$

for all $a>1$ and $N \in \mathbb{N}$. The second sum on the right-hand side of (13) can be upper bounded in the same way.

Proof of Theorem 3. Let $\phi \in \mathcal{M}^{2}(a)$ be arbitrary but fixed. Thus, by Theorem $1, \hat{\phi}(\omega)=1 / a$ a.e. for $|\omega| \leq \pi$. Next, we analyze

$$
\left(H_{N, \phi}^{a} f\right)(t)=\sum_{k=-N}^{N} f\left(\frac{k}{a}\right) \tilde{\phi}\left(t-\frac{k}{a}\right)=\frac{1}{2 \pi} \int_{-\pi}^{\pi} \hat{f}(\omega) \sum_{k=-N}^{N} \mathrm{e}^{i \omega k / a} \tilde{\phi}\left(t-\frac{k}{a}\right) \mathrm{d} \omega .
$$

For the functions $f_{L}, L \in \mathbb{N}$, defined by

$$
\hat{f_{L}}(\omega)= \begin{cases}L \pi & |\omega| \leq \frac{1}{L} \\ 0 & |\omega|>\frac{1}{L}\end{cases}
$$

we obviously have $\left\|f_{L}\right\|_{\mathcal{P} \mathcal{W}_{\pi}^{1}}=1$ for all $L \in \mathbb{N}$. Since

$$
\begin{aligned}
& \lim _{L \rightarrow \infty}\left|\frac{1}{2 \pi} \int_{-\pi}^{\pi} \hat{f}_{L}(\omega) \sum_{k=-N}^{N} \mathrm{e}^{i \omega k / a} \tilde{\phi}\left(t-\frac{k}{a}\right) \mathrm{d} \omega\right| \\
& =\lim _{L \rightarrow \infty}\left|\sum_{k=-N}^{N} \tilde{\phi}\left(t-\frac{k}{a}\right) \frac{L}{2} \int_{-1 / L}^{1 / L} \mathrm{e}^{i \omega k / a} \mathrm{~d} \omega\right| \\
& =\lim _{L \rightarrow \infty}\left|\sum_{k=-N}^{N} \tilde{\phi}\left(t-\frac{k}{a}\right)\left(\frac{a L}{k}\right) \sin \left(\frac{k}{a L}\right)\right|=\left|\sum_{k=-N}^{N} \tilde{\phi}\left(t-\frac{k}{a}\right)\right|,
\end{aligned}
$$

it follows that

$$
\sup _{\|f\|_{\mathcal{P} \mathcal{W}_{\pi}^{1}} \leq 1}\left|\sum_{k=-N}^{N} f\left(\frac{k}{a}\right) \tilde{\phi}\left(t-\frac{k}{a}\right)\right| \geq\left|\sum_{k=-N}^{N} \tilde{\phi}\left(t-\frac{k}{a}\right)\right| .
$$

Moreover,

$$
\begin{aligned}
\tilde{\phi}(t) & =\frac{1}{2 \pi} \int_{-a \pi}^{a \pi}(-i \operatorname{sgn}(\omega)) \hat{\phi}(\omega) \mathrm{e}^{i \omega t} \mathrm{~d} \omega \\
& =\frac{1}{2 \pi a} \int_{-\pi}^{\pi}(-i \operatorname{sgn}(\omega)) \mathrm{e}^{i \omega t} \mathrm{~d} \omega+\underbrace{\frac{1}{2 \pi} \int_{\pi \leq|\omega| \leq a \pi}(-i \operatorname{sgn}(\omega)) \hat{\phi}(\omega) \mathrm{e}^{i \omega t} \mathrm{~d} \omega}_{=q(t)} \\
& =\frac{1}{a \pi} \int_{0}^{\pi} \sin (\omega t) \mathrm{d} \omega+q(t)=\frac{1-\cos (\pi t)}{a \pi t}+q(t) .
\end{aligned}
$$


Note, $q(t) \equiv 0$ for $a=1$. For $t_{N}=\frac{N+1}{a}$ we obtain

$$
\left|\sum_{k=-N}^{N} \tilde{\phi}\left(t_{N}-\frac{k}{a}\right)\right| \geq|\frac{1}{\pi} \underbrace{\sum_{k=-N}^{N} \frac{1-\cos \left(\pi\left(\frac{N+1}{a}-\frac{k}{a}\right)\right)}{a\left(\frac{N+1}{a}-\frac{k}{a}\right)} \mid}_{=L_{N}(a)}-| \sum_{k=-N}^{N} q\left(t_{N}-\frac{k}{a}\right) \mid .
$$

Next the right-hand side of (15) is further analyzed. The first term gives

$$
L_{N}(a)=\sum_{k=-N}^{N} \frac{1-\cos \left(\pi\left(\frac{N+1}{a}-\frac{k}{a}\right)\right)}{a\left(\frac{N+1}{a}-\frac{k}{a}\right)}=\sum_{k=1}^{2 N+1} \frac{1-\cos \left(\frac{\pi}{a} k\right)}{k} .
$$

For $a=1$ this immediately simplifies to

$$
L_{N}(1)=\sum_{k=1}^{2 N+1} \frac{1-\cos (\pi k)}{k}=\sum_{k=0}^{N} \frac{2}{2 k+1} \geq \sum_{k=0}^{N} \int_{k}^{k+1} \frac{1}{\tau+\frac{1}{2}} \mathrm{~d} \tau=\log (2 N+3) .
$$

For $a>1$ a little calculation is needed:

$$
L_{N}(a)=\sum_{k=1}^{2 N+1} \frac{1}{k}-\sum_{k=1}^{2 N+1} \frac{\cos \left(\frac{\pi}{a} k\right)}{k} \geq \log (2 N+2)-\sum_{k=1}^{2 N+1} \frac{\cos \left(\frac{\pi}{a} k\right)}{k}
$$

by the same calculation as in (16). Thus,

$$
\left|L_{N}(a)\right| \geq \log (2 N+2)-\left|\sum_{k=1}^{2 N+1} \frac{\cos \left(\frac{\pi}{a} k\right)}{k}\right| \geq \log (2 N+2)-\frac{1}{\sin \left(\frac{\pi}{2 a}\right)}
$$

by Lemma 3 .

The second term of (15) gives for $a>1$

$$
\begin{aligned}
\sum_{k=-N}^{N} q\left(t_{N}-\frac{k}{a}\right) & =\frac{1}{2 \pi} \int_{\pi \leq|\omega| \leq a \pi}(-i \operatorname{sgn}(\omega)) \hat{\phi}(\omega) \sum_{k=-N}^{N} \mathrm{e}^{i \omega\left(t_{N}-k / a\right)} \mathrm{d} \omega \\
& =\frac{1}{2 \pi} \int_{\pi \leq|\omega| \leq a \pi}(-i \operatorname{sgn}(\omega)) \hat{\phi}(\omega) \mathrm{e}^{i \omega t_{N}} \sum_{k=-N}^{N} \mathrm{e}^{i \omega k / a} \mathrm{~d} \omega .
\end{aligned}
$$

Since

$$
\sum_{k=-N}^{N} \mathrm{e}^{i \omega k / a}=\frac{\mathrm{e}^{-i N \omega / a}-\mathrm{e}^{i(N+1) \omega / a}}{1-\mathrm{e}^{i \omega / a}}
$$


we obtain

$$
\begin{aligned}
\left|\sum_{k=-N}^{N} q\left(t_{N}-\frac{k}{a}\right)\right| & \leq \frac{1}{2 \pi} \int_{\pi \leq|\omega| \leq a \pi}|\hat{\phi}(\omega)| \cdot\left|\frac{\mathrm{e}^{-i N \omega / a}-\mathrm{e}^{i(N+1) \omega / a}}{1-\mathrm{e}^{i \omega / a}}\right| \mathrm{d} \omega \\
& \leq C_{8} \frac{\|\phi\|_{\mathcal{P W}}^{1}}{\left|1-\mathrm{e}^{i \pi / a}\right|} .
\end{aligned}
$$

Therefore, for each $N \in \mathbb{N}$ and $a \geq 1$

$$
\sup _{\|f\|_{\mathcal{P W} \frac{1}{\pi}} \leq 1}\left|\sum_{k=-N}^{N} f\left(\frac{k}{a}\right) \tilde{\phi}\left(t_{N}-\frac{k}{a}\right)\right| \geq \frac{1}{\pi} \log (2 N)-C_{9} .
$$

Hence, by the Banach-Steinhaus theorem there exists a $f_{1} \in \mathcal{P} \mathcal{W}_{\pi}^{1}$ such that

$$
\limsup _{N \rightarrow \infty}\left(\max _{t \in \mathbb{R}}\left|\sum_{k=-N}^{N} f_{1}\left(\frac{k}{a}\right) \tilde{\phi}\left(t-\frac{k}{a}\right)\right|\right)=\infty .
$$

Theorem 3 shows that it is possible to find for all $a \geq 1$ and $\phi \in \mathcal{M}^{2}(a)$ a signal $f_{1} \in \mathcal{P} \mathcal{W}_{\pi}^{1}$, which depends on $\phi$ and $a$, such that the peak value of $H_{N, \phi}^{a} f_{1}$ diverges unboundedly. Immediately the question arises: is there a universal signal $f_{1} \in \mathcal{P} \mathcal{W}_{\pi}^{1}$ such that equation (12) of Theorem 3 holds for all $\phi \in \mathcal{M}^{2}(a)$, $a \geq 1$ ? We will show for a smaller class of approximation processes, namely, all approximation process with $\phi \in \mathcal{M}(a), a>1$, that it is possible to find a universal signal that creates divergence for all approximation processes.

Theorem 4. There exists a signal $f_{1} \in \mathcal{P} \mathcal{W}_{\pi}^{1}$ such that for all $a>1$ and all $\phi \in \mathcal{M}(a)$ we have

$$
\limsup _{N \rightarrow \infty}\left(\sup _{t \in \mathbb{R}}\left|\left(H f_{1}\right)(t)-\left(H_{N, \phi}^{a} f_{1}\right)(t)\right|\right)=\infty .
$$

Remark 5. Note that Theorem 4 is more general than Theorems 2 and 3 in the sense that we have one universal signal such that divergence appears for all approximation processes, whereas in Theorems 2 and 3 the signal can be selected according to the approximation process. However, Theorem 4 does not imply Theorems 2 and 3 because in Theorem 4 the set of permissible approximation processes is smaller: We require $\phi \in \mathcal{M}(a) \subset \mathcal{M}^{2}(a)$.

Remark 6. We want to emphasize that the Hilbert transform is only an example operator, and that there are other translation invariant, linear, and bounded operators - even operators $T$ with continuous $\hat{h}_{T}$ - for which we have divergence. 
Proof of Theorem 4. Let $a>1$ be arbitrary but fixed and $\phi \in \mathcal{M}(a)$. The function $\hat{\phi}$ can be divided into two parts, $\hat{\phi}_{1}$ and $\hat{\phi}_{2}$, by setting $\hat{\phi}_{1}(\omega)=\hat{\phi}(\omega) \hat{w}_{1}(\omega)$ and $\hat{\phi}_{2}(\omega)=\hat{\phi}(\omega) \hat{w}_{2}(\omega)$, where $\hat{w}_{1}(\omega)=\xi_{\pi / 2, \pi}(\omega), \hat{w}_{2}(\omega)=\xi_{a \pi, a \pi+\pi / 2}(\omega)-$ $\xi_{\pi / 2, \pi}(\omega)$, and

$$
\xi_{a, b}(\omega)= \begin{cases}1 & |\omega| \leq a \\ \frac{|\omega|-a}{a-b}+1 & a<|\omega|<b \\ 0 & |\omega| \geq b\end{cases}
$$

Obviously, $w_{1}, w_{2} \in L^{1}, \hat{w}_{1}(\omega)+\hat{w}_{2}(\omega)=1$ for $|\omega| \leq a \pi$ and $\hat{\phi}=\hat{\phi}_{1}+\hat{\phi}_{2}$. Since $\phi_{2}(t)=\int_{-\infty}^{\infty} \phi(\tau) w_{2}(t-\tau) \mathrm{d} \tau$ and

$$
\begin{aligned}
\left\|\phi_{2}\right\|_{1} & \leq \int_{-\infty}^{\infty} \int_{-\infty}^{\infty}|\phi(\tau)|\left|w_{2}(t-\tau)\right| \mathrm{d} \tau \mathrm{d} t \\
& =\int_{-\infty}^{\infty}|\phi(\tau)| \mathrm{d} \tau \int_{-\infty}^{\infty}\left|w_{2}(\tau)\right| \mathrm{d} \tau=\|\phi\|_{1}\left\|w_{2}\right\|_{1}<\infty
\end{aligned}
$$

by Fubini's theorem, we see that $\phi_{2} \in \mathcal{B}_{a \pi}^{1}$.

Moreover, from $-i \operatorname{sgn}(\omega) \hat{\phi}(\omega)=-i \operatorname{sgn}(\omega) \hat{\phi}_{1}(\omega)-i \operatorname{sgn}(\omega) \hat{\phi}_{2}(\omega)$, we obtain $\tilde{\phi}(t)=\tilde{\phi}_{1}(t)+\tilde{\phi}_{2}(t)$ and $\tilde{\phi}_{2} \in \mathcal{B}_{a \pi}^{1}$. Consequently,

$$
\begin{aligned}
& \left|\sum_{k=-N}^{N} f\left(\frac{k}{a}\right) \tilde{\phi}_{2}\left(t-\frac{k}{a}\right)\right| \leq \sum_{k=-N}^{N}\left|f\left(\frac{k}{a}\right)\right| \cdot\left|\tilde{\phi}_{2}\left(t-\frac{k}{a}\right)\right| \\
& \leq\|f\|_{\mathcal{P} \mathcal{W}_{\pi}^{1}} \sum_{k=-N}^{N}\left|\tilde{\phi}_{2}\left(t-\frac{k}{a}\right)\right| \leq C_{10}\|f\|_{\mathcal{P} \mathcal{W}_{\pi}^{1}}\left\|\tilde{\phi}_{2}\right\|_{\mathcal{B}_{a \pi}^{1}},
\end{aligned}
$$

which implies that

$$
\left|\sum_{k=-N}^{N} f\left(\frac{k}{a}\right) \tilde{\phi}\left(t-\frac{k}{a}\right)\right| \geq\left|\sum_{k=-N}^{N} f\left(\frac{k}{a}\right) \tilde{\phi}_{1}\left(t-\frac{k}{a}\right)\right|-C_{10}\|f\|_{\mathcal{P} \mathcal{W}_{\pi}^{1}}\left\|\tilde{\phi}_{2}\right\|_{\mathcal{B}_{a \pi}^{1}} .
$$

It remains to show that there is a $f_{1} \in \mathcal{P} \mathcal{W}_{\pi}^{1}$ such that

$$
\limsup _{N \rightarrow \infty}\left(\sup _{t \in \mathbb{R}}\left|\sum_{k=-N}^{N} f_{1}\left(\frac{k}{a}\right) \tilde{\phi}_{1}\left(t-\frac{k}{a}\right)\right|\right)=\infty
$$

First, we need the Hilbert transform $\tilde{\phi}_{1}$, which can be obtained by evaluating $\tilde{\phi}_{1}(t)=1 /(2 \pi) \int_{-\infty}^{\infty}-i \operatorname{sgn}(\omega) \hat{\phi}_{1}(\omega) \mathrm{e}^{i \omega t} \mathrm{~d} \omega$. A short calculation gives

$$
\tilde{\phi}_{1}(t)=\frac{1}{a \pi t}+\frac{2}{a \pi^{2} t^{2}}\left(\sin \left(\frac{\pi}{2} t\right)-\sin (\pi t)\right) .
$$


The function $\tilde{\phi}_{1}$ has a removable singularity at $t=0$. By setting $\tilde{\phi}_{1}(0)=0$, it extends to a continuous functions on $\mathbb{R}$. Furthermore,

$$
\left|\sin \left(\frac{\pi}{2} t\right)-\sin (\pi t)\right|=\left|2 \cos \left(\frac{3 \pi}{4} t\right) \sin \left(-\frac{\pi}{4} t\right)\right| \leq\left|2 \sin \left(-\frac{\pi}{4} t\right)\right|<\frac{\pi}{2} t,
$$

and therefore

$$
\tilde{\phi}_{1}(t)>0 \text { for } t>0 .
$$

Next, consider the functions

$$
g_{N}(t)=\frac{\sin \left(\frac{t \pi}{N}\right)}{\frac{t \pi}{N}} .
$$

Since

$$
\hat{g}_{N}(\omega)= \begin{cases}N & |\omega| \leq \frac{\pi}{N} \\ 0 & |\omega|>\frac{\pi}{N}\end{cases}
$$

we have $\left\|g_{N}\right\|_{\mathcal{P W}_{\pi}^{1}}=1$ for all $N \in \mathbb{N}$. The functions $g_{N}$ are the building blocks of the universal signal $f_{1}$. We further need a sequence $\left(M_{k}\right)_{k \in \mathbb{N}}$ with the property $M_{k}>2^{\left(k^{3}\right)}$ and $M_{k-1}^{2} \log \left(M_{k}\right) / M_{k}<1, k \in \mathbb{N}$, and define

$$
f_{1}(t)=\sum_{l=1}^{\infty} \frac{1}{l^{2}} g_{M_{l}}(t) .
$$

Since $\left\|f_{1}\right\|_{\mathcal{P W}_{\pi}^{1}} \leq \sum_{l=1}^{\infty}\left(1 / l^{2}\right)\left\|g_{M_{l}}\right\|_{\mathcal{P W}_{\pi}^{1}}=\sum_{l=1}^{\infty} 1 / l^{2}=\pi^{2} / 6$, we have $f_{1} \in$ $\mathcal{P} \mathcal{W}_{\pi}^{1}$.

We divide the expression to be analyzed into three parts, as follows:

$$
\begin{aligned}
\left(H_{M_{r}, \phi_{1}} f_{1}\right)\left(t_{M_{r}}\right)= & \underbrace{\sum_{l=1}^{r-1} \frac{1}{l^{2}}\left(H_{M_{r}, \phi_{1}} g_{M_{l}}\right)\left(t_{M_{r}}\right)}_{=\alpha(r)}+\underbrace{\frac{1}{r^{2}}\left(H_{M_{r}, \phi_{1}} g_{M_{r}}\right)\left(t_{M_{r}}\right)}_{=\beta(r)} \\
& +\underbrace{\sum_{l=r+1}^{\infty} \frac{1}{l^{2}}\left(H_{M_{r}, \phi_{1}} g_{M_{l}}\right)\left(t_{M_{r}}\right)}_{=\gamma(r)},
\end{aligned}
$$

where $r \geq 1$ and $t_{N}=(N+1) / a$, and treat all parts separately.

Part 3: It can be easily seen that

$$
\gamma(r)=\sum_{l=r+1}^{\infty} \frac{1}{l^{2}} \sum_{k=-M_{r}}^{M_{r}} \frac{\sin \left(\frac{k \pi}{a M_{l}}\right)}{\frac{k \pi}{a M_{l}}} \tilde{\phi}_{1}\left(\frac{M_{r}+1-k}{a}\right) \geq 0,
$$

because of $\sin (x) / x \geq 0$ for $|x| \leq \pi$ and equation (20). 
Part 2: Next, we analyze

$$
\beta(r)=\frac{1}{r^{2}} \sum_{k=-M_{r}}^{M_{r}} \frac{\sin \left(\frac{k \pi}{a M_{r}}\right)}{\frac{k \pi}{a M_{r}}} \tilde{\phi}_{1}\left(\frac{M_{r}+1-k}{a}\right) .
$$

By the same argumentation as in Part 3, we have $\beta(r)>0$. Moreover,

$$
\begin{aligned}
\beta(r)= & \frac{1}{r^{2}} \sum_{k=-M_{r}}^{M_{r}} \frac{\sin \left(\frac{k \pi}{a M_{r}}\right)}{\frac{k \pi}{a M_{r}}} \\
& {\left[\frac{1}{a \pi\left(\frac{M_{r}+1-k}{a}\right)}+\frac{2\left(\sin \left(\frac{\pi\left(M_{r}+1-k\right)}{2 a}\right)-\sin \left(\frac{\pi\left(M_{r}+1-k\right)}{a}\right)\right)}{a \pi^{2}\left(\frac{M_{r}+1-k}{a}\right)^{2}}\right] } \\
\geq & \frac{1}{r^{2}} \frac{\sin \left(\frac{\pi}{a}\right)}{\frac{\pi}{a}} \sum_{k=1}^{2 M_{r}+1}\left[\frac{1}{\pi k}+\frac{2 a\left(\sin \left(\frac{\pi k}{2 a}\right)-\sin \left(\frac{\pi k}{a}\right)\right)}{\pi^{2} k^{2}}\right],
\end{aligned}
$$

which can be further simplified by using

$$
\sum_{k=1}^{2 M_{r}+1} \frac{1}{\pi k}>\frac{1}{\pi} \log \left(M_{r}+1\right)>\frac{r^{3}}{\pi} \log (2)
$$

and

$$
\left|\sum_{k=1}^{2 M_{r}+1} \frac{2 a\left(\sin \left(\frac{\pi k}{2 a}\right)-\sin \left(\frac{\pi k}{a}\right)\right)}{\pi^{2} k^{2}}\right| \leq C_{11}
$$

to

$$
\beta(r)>r \frac{\sin \left(\frac{\pi}{a}\right) \log (2)}{\frac{\pi}{a} \pi}-\frac{1}{r^{2}} \frac{\sin \left(\frac{\pi}{a}\right)}{\frac{\pi}{a}} C_{11} .
$$

Obviously,

$$
\lim _{r \rightarrow \infty} \beta(r)=\infty
$$

Part 1: It can be shown that

$$
\lim _{r \rightarrow \infty}|\alpha(r)| \leq C_{12}
$$

with some constant $C_{12}<\infty$. Since the proof of this is rather technical, we put it in the Appendix B.

We have $\left|\left(H_{M_{r}, \phi_{1}} f_{1}\right)\left(t_{M_{r}}\right)\right|=|\alpha(r)+\beta(r)+\gamma(r)| \geq|\beta(r)+\gamma(r)|-|\alpha(r)|$, and since $\beta(r) \geq 0$ and $\gamma(r) \geq 0$ this simplifies to $\left|\left(H_{M_{r}, \phi_{1}} f_{1}\right)\left(t_{M_{r}}\right)\right| \geq \beta(r)-|\alpha(r)|$. Following (21) and (22) we obtain $\lim _{r \rightarrow \infty}\left|\left(H_{M_{r}, \phi_{1}} f_{1}\right)\left(t_{M_{r}}\right)\right|=\infty$, which finishes the proof. 
Theorem 4, where $\phi \in \mathcal{M}(a), a>1$, shows that the global uniform convergence of the Shannon sampling series is destroyed by the linear and bounded operator $H$ in general. In this case, oversampling and a special kernel design do not help to improve the convergence behavior.

Up to this point we have discussed the approximation behavior concerning the supremum norm. We have seen that for the Hilbert transform the approximation process does not converge uniformly on $\mathbb{R}$, even if the original sampling series is uniformly convergent on $\mathbb{R}$. Next, arbitrary operators $T \in \mathcal{L}$ are considered, and it is shown that for all $t_{1} \in \mathbb{R}$ there exists an operator $T \in \mathcal{L}$ and a signal $f_{2} \in \mathcal{P} \mathcal{W}_{\pi}^{1}$ such that the corresponding approximation process diverges for $t_{1}$.

Theorem 5. Let $t_{1} \in \mathbb{R}$ and $\phi \in \mathcal{M}^{2}(a), a \geq 1$. Then there exists an operator $T \in \mathcal{L}$ and a signal $f_{2} \in \mathcal{P} \mathcal{W}_{\pi}^{1}$ such that

$$
\limsup _{N \rightarrow \infty}\left|\left(T f_{2}\right)\left(t_{1}\right)-\left(T_{N, \phi}^{a} f_{2}\right)\left(t_{1}\right)\right|=\infty .
$$

For the proof of Theorem 5 we need Lemma 4.

Lemma 4. For $a>1$ and $N \in \mathbb{N}, N \geq(2 a-1) / 2$, we have

$$
\int_{0}^{\pi / a} \frac{\sin ^{2}\left(\left(N+\frac{1}{2}\right) \omega\right)}{\sin \left(\frac{\omega}{2}\right)} \mathrm{d} \omega>\log \left(\frac{2 N+1}{2 a}-1\right) .
$$

Proof. Let $L_{N, a}$ be the largest natural number such that $\frac{2 L_{N, a} \pi}{2 N+1} \leq \frac{\pi}{a}$, which implies $L_{N, a} \leq \frac{2 N+1}{2 a}$. Then we obtain

$$
\begin{aligned}
& \int_{0}^{\pi / a} \frac{\sin ^{2}\left(\left(N+\frac{1}{2}\right) \omega\right)}{\sin \left(\frac{\omega}{2}\right)} \mathrm{d} \omega \geq \sum_{k=0}^{L_{N, a}-1} \int_{\frac{2 k \pi}{2 N+1}}^{\frac{2(k+1) \pi}{2 N+1}} \frac{\sin ^{2}\left(\left(N+\frac{1}{2}\right) \omega\right)}{\sin \left(\frac{\omega}{2}\right)} \mathrm{d} \omega \\
& >\sum_{k=0}^{L_{N, a}-1} \frac{1}{\sin \left(\frac{(k+1) \pi}{2 N+1}\right)} \int_{\frac{2 k \pi}{2 N+1}}^{\frac{2(k+1) \pi}{2 N+1}} \sin ^{2}\left(\left(N+\frac{1}{2}\right) \omega\right) \mathrm{d} \omega \\
& =\frac{2}{2 N+1} \int_{0}^{\pi} \sin ^{2} \omega \mathrm{d} \omega \sum_{k=0}^{L_{N, a}-1} \frac{1}{\sin \left(\frac{(k+1) \pi}{2 N+1}\right)}>\sum_{k=0}^{L_{N, a}-1} \frac{1}{k+1},
\end{aligned}
$$

where we used $\sin x<x$ for all $x>0$ in the last inequality. But

$$
\sum_{k=0}^{L_{N, a}-1} \frac{1}{k+1} \geq \log \left(L_{N, a}\right)>\log \left(\frac{2 N+1}{2 a}-1\right),
$$

which completes the proof. 
Proof of Theorem 5. Due to the translation invariance of $T \in \mathcal{L}$, we can assume $t_{1}=0$ without loss of generality. Clearly,

$$
\left(T_{N, \phi}^{a} f\right)(0)=\frac{1}{2 \pi} \int_{-\pi}^{\pi} \hat{f}(\omega) \sum_{k=-N}^{N} \mathrm{e}^{i \omega k / a}(T \phi)\left(-\frac{k}{a}\right) \mathrm{d} \omega .
$$

Since $T \phi$ has the representation $(T \phi)(t)=\frac{1}{2 \pi} \int_{-a \pi}^{a \pi} \hat{\phi}(\omega) \hat{h}_{T}(\omega) \mathrm{e}^{i \omega t} \mathrm{~d} \omega$, with some $\hat{h}_{T}(\omega) \in L^{\infty}[-a \pi, a \pi]$, we obtain

$$
\begin{aligned}
\sum_{k=-N}^{N}(T \phi)\left(-\frac{k}{a}\right)= & \frac{1}{2 \pi a} \int_{-\pi}^{\pi} \hat{h}_{T}(\omega) \sum_{k=-N}^{N} \mathrm{e}^{i \omega k / a} \mathrm{~d} \omega \\
& +\underbrace{\frac{1}{2 \pi} \int_{\pi \leq|\omega| \leq a \pi} \hat{h}_{T}(\omega) \hat{\phi}(\omega) \sum_{k=-N}^{N} \mathrm{e}^{i \omega k / a} \mathrm{~d} \omega}_{=: R_{N}} \\
= & \frac{1}{2 \pi a} \int_{-\pi}^{\pi} \hat{h}_{T}(\omega) \frac{\sin \left(\left(N+\frac{1}{2}\right) \frac{\omega}{a}\right)}{\sin \left(\frac{\omega}{2 a}\right)} \mathrm{d} \omega+R_{N},
\end{aligned}
$$

where we identified $\sum_{k=-N}^{N} \mathrm{e}^{i \omega k / a}$ as the Dirichlet kernel. Using (18), the modulus of $R_{N}$ can be bounded above independently of $N$ by

$$
\left|R_{N}\right| \leq C_{13} \frac{\|\hat{\phi}\|_{\infty}\left\|\hat{h}_{T}\right\|_{\infty}}{\left|1-\mathrm{e}^{i \pi / a}\right|}
$$

We use the test function

$$
\hat{g}_{N}(\omega)=\sin \left(\left(N+\frac{1}{2}\right) \frac{\omega}{a}\right) \hat{g}(\omega),
$$

where $\hat{g}$ is an even, continuous function with $\hat{g}(\omega)=1,0 \leq|\omega| \leq \pi$ and $\hat{g}(\omega)=0$, $|\omega| \geq a \pi$. Then, using Lemma 4 , we obtain

$$
\begin{aligned}
& \frac{1}{2 \pi a} \int_{-\pi}^{\pi} \hat{g}_{N}(\omega) \frac{\sin \left(\left(N+\frac{1}{2}\right) \frac{\omega}{a}\right)}{\sin \left(\frac{\omega}{2 a}\right)} \mathrm{d} \omega=\frac{1}{\pi a} \int_{0}^{\pi} \frac{\sin ^{2}\left(\left(N+\frac{1}{2}\right) \frac{\omega}{a}\right)}{\sin \left(\frac{\omega}{2 a}\right)} \mathrm{d} \omega \\
& =\frac{1}{\pi} \int_{0}^{\pi / a} \frac{\sin ^{2}\left(\left(N+\frac{1}{2}\right) \omega\right)}{\sin \left(\frac{\omega}{2}\right)} \mathrm{d} \omega>\frac{1}{\pi} \log \left(\frac{2 N+1}{2 a}-1\right)
\end{aligned}
$$

for $N \geq(2 a-1) / 2$. By the Banach-Steinhaus theorem there exists a function $\hat{h}_{T}$ such that

$$
\limsup _{N \rightarrow \infty}\left|\frac{1}{2 \pi a} \int_{-\pi}^{\pi} \hat{h}_{T}(\omega) \frac{\sin \left(\left(N+\frac{1}{2}\right) \frac{\omega}{a}\right)}{\sin \left(\frac{\omega}{2 a}\right)} \mathrm{d} \omega\right|=\infty .
$$


Since

$$
\limsup _{N \rightarrow \infty} \sup _{\|f\|_{\mathcal{P} \mathcal{W}_{\pi}^{1}} \leq 1}\left|\left(T_{N, \phi}^{a} f\right)(0)\right| \geq \limsup _{N \rightarrow \infty}\left|\sum_{k=-N}^{N}(T \phi)\left(-\frac{k}{a}\right)\right|=\infty,
$$

we can again apply the Banach-Steinhaus theorem, which states the existence of a signal $f_{2} \in \mathcal{P} \mathcal{W}_{\pi}^{1}$ such that $\lim \sup _{N \rightarrow \infty}\left|\left(T_{N, \phi}^{a} f_{2}\right)(0)\right|=\infty$.

We have seen that it is not possible to stably approximate the Hilbert transform on the basis of the samples $\{f(k / a)\}_{k \in \mathbb{Z}}, a \geq 1$ in general. The size of a is irrelevant, i.e., the result is valid for all $a \geq 1$. This shows that a stable sampling-based signal approximation cannot be realized in general.

However, this does not mean that a stable computation of $\tilde{f}$ from the signal values $\{f(t)\}_{t \in \mathbb{R}}$ is impossible. Consider

$$
\left(H_{\epsilon} f\right)(t)=\frac{1}{\pi} \int_{\epsilon \leq|\tau| \leq \frac{1}{\epsilon}} \frac{1}{\tau} f(t+\tau) \mathrm{d} \tau .
$$

Theorem 6. For all $f \in \mathcal{P} \mathcal{W}_{\pi}^{1}$ we have $\lim _{\epsilon \rightarrow 0}\left\|\tilde{f}-H_{\epsilon} f\right\|_{\infty}=0$.

Proof. Let $f \in \mathcal{P} \mathcal{W}_{\pi}^{1}$ and $\delta>0$ arbitrary but fixed. Then we have

$$
\begin{aligned}
\left|\tilde{f}(t)-\left(H_{\epsilon} f\right)(t)\right| & =\left|\frac{1}{2 \pi} \int_{-\pi}^{\pi} \hat{f}(\omega) \mathrm{e}^{i \omega t}\left(-i \operatorname{sgn}(\omega)+\frac{1}{\pi} \int_{\epsilon \leq|\tau| \leq \frac{1}{\epsilon}} \frac{1}{\tau} \mathrm{e}^{i \omega \tau} \mathrm{d} \tau\right) \mathrm{d} \omega\right| \\
& \leq \frac{1}{2 \pi} \int_{-\pi}^{\pi}|\hat{f}(\omega)|\left|\operatorname{sgn}(\omega)-\frac{2}{\pi} \int_{\epsilon}^{1 / \epsilon} \frac{\sin (\omega \tau)}{\tau} \mathrm{d} \tau\right| \mathrm{d} \omega
\end{aligned}
$$

and

$$
\left|\frac{2}{\pi} \int_{\epsilon}^{1 / \epsilon} \frac{\sin (\omega \tau)}{\tau} \mathrm{d} \tau\right| \leq\left|\frac{2}{\pi} \int_{0}^{1} \frac{\sin (\tau)}{\tau} \mathrm{d} \tau\right| \leq C_{14}
$$

for all $\epsilon>0$ and $\omega \in \mathbb{R}$. There exists a $\omega_{0}=\omega_{0}(\delta)<\pi$ such that

$$
\frac{1}{2 \pi} \int_{-\omega_{0}}^{\omega_{0}}|\hat{f}(\omega)| \mathrm{d} \omega<\delta
$$

and

$$
\frac{1}{2 \pi} \int_{-\omega_{0}}^{\omega_{0}}|\hat{f}(\omega)| \operatorname{sgn}(\omega)-\frac{2}{\pi} \int_{\epsilon}^{1 / \epsilon} \frac{\sin (\omega \tau)}{\tau} \mathrm{d} \tau \mid \mathrm{d} \omega<\delta\left(1+C_{14}\right)
$$

for all $\epsilon>0$. Moreover, there exists a $\epsilon_{0}=\epsilon_{0}(\delta)$ such that

$$
\left|1-\frac{2}{\pi} \int_{\epsilon}^{1 / \epsilon} \frac{\sin (\omega \tau)}{\tau} \mathrm{d} \tau\right|<\frac{\delta}{2}
$$


for all $0<\epsilon \leq \epsilon_{0}$ and $\omega \in\left[\omega_{0}, \pi\right]$. Consequently, for all $0<\epsilon \leq \epsilon_{0}$

$$
\begin{aligned}
\left|\tilde{f}(t)-\left(H_{\epsilon} f\right)(t)\right| \leq & \frac{1}{2 \pi} \int_{-\omega_{0}}^{\omega_{0}}|\hat{f}(\omega)|\left|\operatorname{sgn}(\omega)-\frac{2}{\pi} \int_{\epsilon}^{1 / \epsilon} \frac{\sin (\omega \tau)}{\tau} \mathrm{d} \tau\right| \mathrm{d} \omega \\
& +\frac{1}{2 \pi} \int_{\omega_{0} \leq|\omega| \leq \pi}|\hat{f}(\omega)|\left|\operatorname{sgn}(\omega)-\frac{2}{\pi} \int_{\epsilon}^{1 / \epsilon} \frac{\sin (\omega \tau)}{\tau} \mathrm{d} \tau\right| \mathrm{d} \omega \\
\leq & \delta\left(1+C_{14}\right)+\delta \frac{1}{2 \pi} \int_{\omega_{0} \leq|\omega| \leq \pi}|\hat{f}(\omega)| \mathrm{d} \omega \leq \delta\left(1+C_{14}\right)+\delta\|f\|_{\mathcal{P} \mathcal{W}_{\pi}^{1}}
\end{aligned}
$$

Since $\delta$ was arbitrary, the proof is complete.

\section{$5 \quad$ Test Signals}

The results in Section 4 reveal the very intricate convergence behavior of the approximation processes. We have shown that there is no universal approximation process of the shape (11) that is convergent for all operators $T \in \mathcal{L}$ and all signals $f \in \mathcal{P} \mathcal{W}_{\pi}^{1}$. Furthermore, we have seen that the Hilbert transform $H \in \mathcal{L}$ is a universal operator in the sense that for every kernel $\phi \in \mathcal{M}^{2}(a)$ there exist a signal $f_{1} \in \mathcal{P} \mathcal{W}_{\pi}^{1}$ such that

$$
\limsup _{N \rightarrow \infty}\left(\sup _{t \in \mathbb{R}}\left|\left(H_{N, \phi}^{a} f_{1}\right)(t)\right|\right)=\infty
$$

In other words, there is no uniformly convergent approximation process for the Hilbert transform. However, for other operators a uniformly convergent approximation process can exist. It would be useful to have a simple criterion whether an approximation process is convergent for a given operator or not. In this section we will provide a simple test for convergence, which uses the exponential function, namely, $f_{\omega}^{\text {test }}(t)=\mathrm{e}^{i \omega t},-\pi \leq \omega \leq \pi$, as test signal. Note the simple structure of the test signals: They are scaled versions of one basic function $\mathrm{e}^{i t}$. Obviously, the set of test signals is neither dense in $\mathcal{P} \mathcal{W}_{\pi}^{1}$ nor does it form a linear space.

Theorem 7. For all $a \geq 1, \phi \in \mathcal{M}^{2}(a), t \in \mathbb{R}$ and $T \in \mathcal{L}\left(\mathcal{P} \mathcal{W}_{a \pi}^{1}\right)$ we have:

$$
\lim _{N \rightarrow \infty}\left(T_{N, \phi}^{a} f\right)(t)=(T f)(t)
$$

for all $f \in \mathcal{P} \mathcal{W}_{\pi}^{1}$ if and only if there exists a constant $C_{15}=C_{15}(t)$ such that

$$
\max _{|\omega| \leq \pi}\left|\sum_{k=-N}^{N} \mathrm{e}^{i \omega k / a}(T \phi)\left(t-\frac{k}{a}\right)\right| \leq C_{15}(t)
$$

for all $N \in \mathbb{N}$. 
Proof. First part, " $\Rightarrow$ ": Let $t \in \mathbb{R}$ be arbitrary but fixed. Then, by assumption, $\lim _{N \rightarrow \infty}\left|(T f)(t)-\left(T_{N, \phi}^{a} f\right)(t)\right|=0$ for all $f \in \mathcal{P} \mathcal{W}_{\pi}^{1}$, which implies $\sup _{N \in \mathbb{N}} \sup _{\|f\|_{\mathcal{P W} \text { 市 }} \leq 1}\left|\left(T_{N, \phi}^{a} f\right)(t)\right|<\infty$. This together with

$$
\sup _{\|f\|_{\mathcal{P} \mathcal{W}_{\pi}^{1} \leq 1}}\left|\left(T_{N, \phi}^{a} f\right)(t)\right|=\max _{|\omega| \leq \pi}\left|\sum_{k=-N}^{N} \mathrm{e}^{i \omega k / a}(T \phi)\left(t-\frac{k}{a}\right)\right|
$$

completes the first part.

Second part, " $\Leftarrow$ ": Let $t \in \mathbb{R}$ be arbitrary but fixed. For each $\epsilon>0$ there exists a $g \in \mathcal{P} \mathcal{W}_{\pi}^{2}$ such that $\|f-g\|_{\mathcal{P W}_{\pi}^{1}}<\epsilon$ and consequently $\mid(T f)(t)-$ $(T g)(t) \mid<\epsilon\|T\|_{\mathcal{L}\left(\mathcal{P} \mathcal{W}_{a \pi}^{1}\right)}$. Obviously, we have

$$
\begin{aligned}
\mid(T f)(t) & -\left(T_{N, \phi}^{a} f\right)(t) \mid \\
& =\left|(T f)(t)-(T g)(t)+(T g)(t)-\left(T_{N, \phi}^{a} g\right)(t)+\left(T_{N, \phi}^{a}(g-f)\right)(t)\right| \\
& \leq|(T f)(t)-(T g)(t)|+\left|(T g)(t)-\left(T_{N, \phi}^{a} g\right)(t)\right|+\left|\left(T_{N, \phi}^{a}(g-f)\right)(t)\right| .
\end{aligned}
$$

Furthermore,

$$
\begin{aligned}
\left|\left(T_{N, \phi}^{a}(g-f)\right)(t)\right| & =\left|\frac{1}{2 \pi} \int_{-\pi}^{\pi}(\hat{g}(\omega)-\hat{f}(\omega)) \sum_{k=-N}^{N} \mathrm{e}^{i \omega k / a}(T \phi)\left(t-\frac{k}{a}\right) \mathrm{d} \omega\right| \\
& \leq \frac{1}{2 \pi} \int_{-\pi}^{\pi}|\hat{g}(\omega)-\hat{f}(\omega)|\left|\sum_{k=-N}^{N} \mathrm{e}^{i \omega k / a}(T \phi)\left(t-\frac{k}{a}\right)\right| \mathrm{d} \omega \\
& \leq \max _{|\omega| \leq \pi}\left|\sum_{k=-N}^{N} \mathrm{e}^{i \omega k / a}(T \phi)\left(t-\frac{k}{a}\right)\right|\|g-f\|_{\mathcal{P} \mathcal{W}_{\pi}^{1}} \\
& \leq C_{15}(t) \epsilon
\end{aligned}
$$

where we used the assumption in the last inequality. Moreover, there exists a $N_{0}=N_{0}(\epsilon)$ such that $\left|(T g)(t)-\left(T_{N, \phi}^{a} g\right)(t)\right|<\epsilon$ for all $N \geq N_{0}$. Therefore, $\left|(T f)(t)-\left(T_{N, \phi}^{a} f\right)(t)\right|<\left(1+\|T\|_{\mathcal{L}\left(\mathcal{P} \mathcal{W}_{a \pi}^{1}\right)}+C_{15}(t)\right) \epsilon$ for all $N \geq N_{0}$ and the proof is complete because $\epsilon$ was arbitrary.

Theorem 8. For all $a \geq 1, \phi \in \mathcal{M}^{2}(a)$ and $T \in \mathcal{L}\left(\mathcal{P} \mathcal{W}_{a \pi}^{1}\right)$ we have:

$$
\lim _{N \rightarrow \infty}\left\|T f-T_{N, \phi}^{a} f\right\|_{\infty}=0
$$

for all $f \in \mathcal{P} \mathcal{W}_{\pi}^{1}$ if and only if there exists a constant $C_{16}$, independently of $t$, such that

$$
\sup _{t \in \mathbb{R}} \max _{|\omega| \leq \pi}\left|\sum_{k=-N}^{N} \mathrm{e}^{i \omega k / a}(T \phi)\left(t-\frac{k}{a}\right)\right| \leq C_{16}
$$

for all $N \in \mathbb{N}$ and $t \in \mathbb{R}$. 
Proof. Analogously to the proof of Theorem 7.

Corollary 1. For all $a \geq 1, \phi \in \mathcal{M}^{2}(a)$ and $t \in \mathbb{R}$ we have:

$$
\lim _{N \rightarrow \infty}\left(A_{N, \phi}^{a} f\right)(t)=f(t)
$$

for all $f \in \mathcal{P} \mathcal{W}_{\pi}^{1}$ if and only if for all $t \in \mathbb{R}$ there exists a constant $C_{17}=C_{17}(t)$ such that

$$
\max _{|\omega| \leq \pi}\left|\sum_{k=-N}^{N} \mathrm{e}^{i \omega k / a} \phi\left(t-\frac{k}{a}\right)\right| \leq C_{17}(t)
$$

for all $N \in \mathbb{N}$.

Proof. Corollary 1 is a special case of Theorem 7 with $T=I d$.

The next corollary is similar to Corollary 1 but makes a statement about the uniform convergence of $A_{N, \phi}^{a} f$.

Corollary 2. For all $a \geq 1$ and $\phi \in \mathcal{M}^{2}(a)$ we have:

$$
\lim _{N \rightarrow \infty}\left\|f-A_{N, \phi}^{a} f\right\|_{\infty}=0
$$

for all $f \in \mathcal{P} \mathcal{W}_{\pi}^{1}$ if and only if there exists a constant $C_{18}$, independently of $t$, such that

$$
\sup _{t \in \mathbb{R}} \max _{|\omega| \leq \pi}\left|\sum_{k=-N}^{N} \mathrm{e}^{i \omega k / a} \phi\left(t-\frac{k}{a}\right)\right| \leq C_{18}
$$

for all $N \in \mathbb{N}$ and $t \in \mathbb{R}$.

Proof. Corollary 2 is a special case of Theorem 8 with $T=I d$.

The equations (23), (24), (25), and (26) have a pleasant interpretation. The exponential function

$$
f_{\omega}^{\text {test }}(t)=\mathrm{e}^{i \omega t}
$$

can be thought of as a test signal, where the parameter $\omega$ ranges from $-\pi$ to $\pi$. Thus, if for all $t \in \mathbb{R}$ and $N \in \mathbb{N}$

$$
\max _{|\omega| \leq \pi}\left|\sum_{k=-N}^{N} f_{\omega}^{\text {test }}\left(\frac{k}{a}\right) \phi\left(t-\frac{k}{a}\right)\right| \leq C_{17}(t),
$$

then the sampling series $A_{N, \phi}^{a} f(t)$ converges to $f(t)$ for all $f \in \mathcal{P} \mathcal{W}_{\pi}^{1}$ and $t \in \mathbb{R}$.

However the converse statement might be more practicable: If we find one test signal $f_{\omega_{1}}^{\text {test }}$ with some $\omega_{1} \in[-\pi, \pi]$ such that

$$
\lim _{N \rightarrow \infty} \sup _{t \in \mathbb{R}}\left|\sum_{k=-N}^{N} f_{\omega_{1}}^{\text {test }}\left(\frac{k}{a}\right) \phi\left(t-\frac{k}{a}\right)\right|=\infty,
$$


it follows by Corollary 2 that there is a signal $f_{1} \in \mathcal{P} \mathcal{W}_{\pi}^{1}$ such that $A_{N, \phi}^{a} f_{1}$ does not converge uniformly.

We can illustrate the preceding corollaries, i.e., the case $T=I d$, by applying them on the well-known Shannon sampling series.

Example 4. The sampling series $A_{N, \phi}^{a} f$ with $a=1$ and

$$
\phi(t)=\frac{\sin (\pi t)}{\pi t}
$$

is nothing but the Shannon sampling series. For $t \in \mathbb{R}$ fixed we have

$$
\left|\sum_{k=-N}^{N} f_{\omega}^{\text {test }}(k) \frac{\sin (\pi(t-k))}{\pi(t-k)}\right| \leq C_{17}(t)
$$

for all $\omega \leq \pi$. Thus, by Corollary 1 , the Shannon sampling series converges pointwise to $f$ for all $f \in \mathcal{P} \mathcal{W}_{\pi}^{1}$.

On the other hand we have

$$
\left|\sum_{k=-N}^{N} f_{\pi}^{\text {test }}(k) \frac{\sin \left(\pi\left(t_{N}-k\right)\right)}{\pi\left(t_{N}-k\right)}\right| \geq C_{19} \log N
$$

for $t_{N}=N+1 / 2$, which shows that the Shannon sampling series is not uniformly convergent on $\mathbb{R}$ for $f \in \mathcal{P} \mathcal{W}_{\pi}^{1}$ in general.

Remark 7. From the proof of Theorem 7 and Example 4 it can be seen that the Shannon sampling series is uniformly convergent on all intervals $[-T, T], T>0$, because

$$
\sup _{[-T, T]}\left|\sum_{k=-N}^{N} f_{\omega}^{\text {test }}(k) \frac{\sin (\pi(t-k))}{\pi(t-k)}\right| \leq C_{20}
$$

for all $N \in \mathbb{N}$. Thus, Brown's theorem, namely, the uniform convergence of the Shannon sampling series on all intervals $[-T, T], T>0$, can be derived from test signal criterion.

Example 5. Another example is the Shannon sampling series with oversampling, i.e., $A_{N, \phi}^{a} f$ with $a>1$ and

$$
\phi(t)=\frac{\sin \left(a \pi\left(t-\frac{k}{a}\right)\right)}{a \pi\left(t-\frac{k}{a}\right)} .
$$

Here we have, after a short calculation, for all $|\omega| \leq \pi$ and $t \in \mathbb{R}$ the upper bound

$$
\left|\sum_{k=-N}^{N} f_{\omega}^{\text {test }}\left(\frac{k}{a}\right) \frac{\sin \left(a \pi\left(t-\frac{k}{a}\right)\right)}{a \pi\left(t-\frac{k}{a}\right)}\right| \leq 2\left(1+\frac{2}{\cos \left(\frac{\pi}{2 a}\right)}\right),
$$


which implies, according to Corollary 2, that the Shannon sampling series with oversampling is uniformly convergent on $\mathbb{R}$ for all $f \in \mathcal{P} \mathcal{W}_{\pi}^{1}$.

This example gives a nice interpretation for the mechanisms of oversampling for the Shannon sampling series. If oversampling with $a>1$ is applied, the critical Nyquist frequency is no more $\pi$ but $a \pi$. Thus, the test signals $f_{\omega}^{\text {test }}$, $|\omega| \leq \pi$, do not lead to a divergent series.

Next, we have a look on general approximation processes.

Example 6. For $a \geq 1$ and $\phi \in \mathcal{M}(a)$ we have

$$
\left|\sum_{k=-N}^{N} f_{\omega}^{\text {test }}\left(\frac{k}{a}\right) \phi\left(t-\frac{k}{a}\right)\right| \leq\left\|f_{\omega}^{\text {test }}\right\|_{\infty} \sum_{k=-N}^{N}\left|\phi\left(t-\frac{k}{a}\right)\right| \leq C_{4},
$$

because of Lemma 1 and $\left\|f_{\omega}^{\text {test }}\right\|_{\infty}=1$.

Thus, the uniform convergence of the corresponding sampling series follows immediately by Corollary 2 .

Example 7. Finally, we investigate the convergence behavior of the approximation process $T_{N, \phi}^{a} f$ with $T=D$, where $D$ is the differential operator, and $\phi \in \mathcal{M}^{2}(a)$.

Fist, we analyze the case $a=1$. Then the only possible $\phi$ is $\phi_{1}(t)=$ $\sin (\pi t) /(\pi t)$ and we obtain

$$
\left(D \phi_{1}\right)(t)=\frac{\pi^{2} t \cos (\pi t)-\pi \sin (\pi t)}{(\pi t)^{2}}
$$

and

$$
\begin{aligned}
\left(D_{N, \phi_{1}}^{1} f\right)(N+1) & =\sum_{k=-N}^{N} f(k) \frac{\cos (\pi(N+1-k))}{N+1-k} \\
& =\sum_{k=-N}^{N} f(k) \frac{(-1)^{N+1-k}}{N+1-k} .
\end{aligned}
$$

For the test signal $f_{\pi}^{\text {test }}$ we have $f_{\pi}^{\text {test }}(k)=(-1)^{k}$ and

$$
\left|\left(D_{N, \phi_{1}}^{1} f_{\pi}^{\text {test }}\right)(N+1)\right|=\sum_{k=-N}^{N} \frac{1}{N+1-k} \geq \log (2 N+2) .
$$

Thus,

$$
\lim _{N \rightarrow \infty} \sup _{t \in \mathbb{R}} \max _{|\omega| \leq \pi}\left|\left(D_{N, \phi_{1}}^{1} f_{\omega}^{\text {test }}\right)(t)\right|=\infty
$$


and Theorem 8 shows that there exists a signal in $f_{1} \in \mathcal{P} \mathcal{W}_{\pi}^{1}$ such that $D_{N, \phi_{1}}^{1} f_{1}$ does not converge uniformly on $\mathbb{R}$ to $D f_{1}$. Hence, for $a=1$ there exists no approximation process that converges uniformly on $\mathbb{R}$ to $D f$ for all $f \in \mathcal{P} \mathcal{W}_{\pi}^{1}$.

On the other hand for $a>1$ and $\phi \in \mathcal{M}(a)$, we have $D \phi \in \mathcal{B}_{a \pi}^{1}$ and

$$
\begin{aligned}
\left(D_{N, \phi}^{a} f_{\omega}^{\text {test }}\right)(t) & =\left|\sum_{k=-N}^{N} f_{\omega}^{\text {test }}\left(\frac{k}{a}\right)(D \phi)\left(t-\frac{k}{a}\right)\right| \\
& \leq\left\|f_{\omega}^{\text {test }}\right\|_{\infty} \sum_{k=-N}^{N}\left|(D \phi)\left(t-\frac{k}{a}\right)\right| \\
& \leq C_{4},
\end{aligned}
$$

by Lemma 1 and $\left\|f_{\omega}^{\text {test }}\right\|_{\infty}=1$. Thus,

$$
\sup _{t \in \mathbb{R}} \max _{|\omega| \leq \pi}\left|\left(D_{N, \phi}^{a} f_{\omega}^{\text {test }}\right)(t)\right| \leq C_{4}
$$

for all $N \in \mathbb{N}$ and Theorem 8 shows that $D_{N, \phi}^{a} f$ converges uniformly on $\mathbb{R}$ to $D f$ for $a>1$ and $\phi \in \mathcal{M}(a)$. Since $\mathcal{M}(a) \subset \mathcal{M}^{2}(a), a>1$, there exists an approximation process that converges uniformly on $\mathbb{R}$ to $D f$ for all $f \in \mathcal{P} \mathcal{W}_{\pi}^{1}$.

This example demonstrates that oversampling combined with the choice of a suitable kernel is eligible to improve the convergence behavior for the differential operator.

\section{Conclusions}

We have shown that there is no universal approximation process of the shape (11) that is convergent for all operators $T \in \mathcal{L}$ and all signals $f \in \mathcal{P} \mathcal{W}_{\pi}^{1}$. Furthermore, we have seen that the Hilbert transform $H \in \mathcal{L}$ is a universal operator in the sense that for every kernel $\phi \in \mathcal{M}^{2}(a)$ there exist a signal $f_{1} \in \mathcal{P} \mathcal{W}_{\pi}^{1}$ such that

$$
\limsup _{N \rightarrow \infty}\left(\sup _{t \in \mathbb{R}}\left|\left(H_{N, \phi}^{a} f_{1}\right)(t)\right|\right)=\infty .
$$

In other words, there is no uniformly convergent approximation process for the Hilbert transform. In general, it is not possible to approximate an operator, acting on continuous-time bandlimited functions, by an approximation process that uses only the samples of the function, and even oversampling does not lead to a better convergence behavior. In fact, the convergence has to be checked from case to case. In order to be able to decide whether an approximation process is convergent for a given operator, we derived a simple criterion, which uses the exponential function as test signal. 


\section{ACKNOWLEDGEMENTS}

This work was partly supported by the German Research Foundation (DFG) under grant BO 1734/9-1.

The material in this paper was presented in part at the 2008 IEEE International Symposium on Information Theory [3].

\section{References}

[1] Holger Boche and Ullrich J. Mönich. Behavior of Shannon's sampling series with applications, Proceedings of the 2007 IEEE International Symposium on Information Theory, 301-305, June 2007.

[2] _ Characterization of the reconstruction behavior of generalized sampling series for bandlimited Paley-Wiener functions, Proceedings of European Signal Processing Conference (EUSIPCO), 1980-1984, September 2007.

[3] _ General behavior of sampling-based signal and system representation, Proceedings of the 2008 IEEE International Symposium on Information Theory, pp. 2439-2443, July 2008.

[4] P. L. Butzer, W. Engels, S. Ries, and R. L. Stens.The Shannon sampling series and the reconstruction of signals in terms of linear, quadratic and cubic splines, SIAM Journal on Applied Mathematics, 46(2), 299-323, 1986.

[5] P. L. Butzer, S. Ries, and R. L. Stens. Approximation of continuous and discontinuous functions by generalized sampling series, Journal of Approximation Theory, 50(1), 25-39, 1987.

[6] P. L. Butzer and R. L. Stens. A modification of the Whittaker-KotelnikovShannon sampling series, Aequationes Mathematicae, 28 (1), 305-311, 1985.

[7] L. L. Campbell. Sampling theorem for the Fourier transform of a distribution with bounded support, SIAM Journal on Applied Mathematics, 16 (3), 626-636, 1968.

[8] Zoran Cvetković and Ingrid Daubechies. Single-bit oversampled A/D conversion with exponential accuracy inthe bit-rate, Data Compression Conference (DCC 2000), 343-352, March 2000.

[9] W. Engels, E. L. Stark, and L. Vogt. Optimal kernels for a general sampling theorem, Journal of Approximation Theory, 50 (1), 69-83, 1987. 
[10] Richard P. Feynman. Feynman lectures on computation, Penguin Books, 1999.

[11] John R. Higgins. Five short stories about the cardinal series, -it Bull. Amer. Math. Soc., 12(1), 45-89, 1985.

[12] John R. Higgins and Rudolf. L. Stens (eds.). Sampling theory in Fourier and signal analysis - advanced topics, Oxford University Press, 1999.

[13] D. Jagerman, Bounds for truncation error of the sampling expansion. SIAM Journal on Applied Mathematics, 14(4), 714-723, 1966.

[14] Abdul J. Jerri. The Shannon sampling theorem-its various extensions and applications: A tutorial review, Proceedings of the IEEE, 65(11), 15651596, 1977.

[15] V. A. Kotel'nikov. O propusknoj sposobnosti 'efira' i provoloki v elektrosvjazi, Procs. of the first All-Union Conference on the technological reconstruction of the communications sector and low-current engineering, pp. 119, 1933.

[16] Robert J. Marks II. Introduction to Shannon sampling and interpolation theory, Springer-Verlag, 1991.

[17] Robert J. Marks II (ed.). Advanced topics in Shannon sampling and interpolation theory, Springer-Verlag, 1993.

[18] Farokh Marvasti (ed.). Nonuniform sampling: Theory and practice, Kluwer Academic / Plenum Publishers, 2001.

[19] Ernst Pfaffelhuber. Sampling series for band-limited generalized functions, IEEE Trans. Info. Th., 17(6), 650-654, 1971.

[20] M. Plancherel and G. Pólya. Fonctions entières et intégrales de Fourier multiples, Commentarii Mathematici Helvetici, 9(1), 224-248, 1937.

[21] Liwen Qian. On the regularized Whittaker-Kotel'nikov-Shannon sampling formula, Proceedings of the American Mathematical Society, 131(4), 11691176, 2003.

[22] . Localization operator based on sampling multipliers, Applied and Computational Harmonic Analysis 22 (2007), no. 2, 217-234.

[23] Herbert Raabe. Untersuchungen an der wechselzeitigen Mehrfachübertragung (Multiplexübertragung), Elektrische Nachrichtentechnik, 16(8), 213228, 1939 .

[24] Walter Rudin. Real and complex analysis, 3 ed., McGraw-Hill, 1987. 
[25] Gerhard Schmeisser and Frank Stenger. Sinc approximation with a Gaussian multiplier, Sampl. Theory Signal Image Process., 6(2), 199-221, 2007.

[26] Claude E. Shannon. Communication in the presence of noise, Proceedings of the IEEE, 72 (9), 1192-1201, 1984, Reprinted from the Proceedings of the IRE, vol. 37, no. 1, Jan. 1949, pp. 10-21.

[27] Hans Triebel. Theory of function spaces, Mongraphs in mathematics, vol. 78, Birkhäuser Verlag, 1983.

[28] Michael Unser. Sampling-50 years after Shannon, Proceedings of the IEEE, 88(4), 569-587, 2000.

[29] E. T. Whittaker. On the functions which are represented by the expansions of the interpolation theory, Proceedings of the Royal Society of Edinburgh, 35, 181-194, 1915.

[30] Moshe Zakai. Band-limited functions and the sampling theorem, Information and Control, 8(2), 143-158, 1965.

[31] A. Zygmund. Trigonometric series, 3 ed., vol. I, Cambridge University Press, 2002.

\section{APPENDIX}

\section{A Proof of Observation 2}

Let $T \in \mathcal{L}$ be arbitrary but fixed.

We first prove the existence of such a function. Since $T f \in \mathcal{W}$ for all $f \in \mathcal{W}$, we have

$$
|(T f)(0)| \leq \frac{1}{2 \pi} \int_{-\infty}^{\infty}|(\widehat{T f})(\omega)| \mathrm{d} \omega=\|T f\|_{\mathcal{W}} \leq\|T\|_{\mathcal{L}}\|f\|_{\mathcal{W}}=\frac{1}{2 \pi}\|T\|_{\mathcal{L}}\|\hat{f}\|_{L^{1}(\mathbb{R})}
$$

for all $f \in \mathcal{W}$. Thus, $\Psi: L^{1}(\mathbb{R}) \rightarrow \mathbb{C}, \hat{f} \mapsto(T f)(0)$ is a bounded linear functional. It follows that there exists a function $\hat{h} \in L^{\infty}(\mathbb{R})$ such that

$$
(T f)(0)=\frac{1}{2 \pi} \int_{-\infty}^{\infty} \hat{f}(\omega) \hat{h}(\omega) \mathrm{d} \omega
$$

for all $\hat{f} \in L^{1}(\mathbb{R})$ and, hence, for all $f \in \mathcal{W}$. Let $f_{t}(\tau)=f(\tau-t)$. Then we have

$$
(T f)(t)=\left(T f_{-t}\right)(0)=\frac{1}{2 \pi} \int_{-\infty}^{\infty} \widehat{f_{-t}}(\omega) \hat{h}(\omega) \mathrm{d} \omega=\frac{1}{2 \pi} \int_{-\infty}^{\infty} \hat{f}(\omega) \mathrm{e}^{i \omega t} \hat{h}(\omega) \mathrm{d} \omega
$$

for all $f \in \mathcal{W}$. Thus, $\hat{h}$ is the desired function $\hat{h}_{T}$. 
Next, we show the uniqueness of the function. Suppose that there are two functions $\hat{h}_{1} \in L^{\infty}(\mathbb{R})$ and $\hat{h}_{2} \in L^{\infty}(\mathbb{R})$ such that $(3)$ is true for all $f \in \mathcal{W}$. Then we have

$$
\frac{1}{2 \pi} \int_{-\infty}^{\infty} \hat{h}_{1}(\omega) \hat{f}(\omega) \mathrm{e}^{i \omega t} \mathrm{~d} \omega=\frac{1}{2 \pi} \int_{-\infty}^{\infty} \hat{h}_{2}(\omega) \hat{f}(\omega) \mathrm{e}^{i \omega t} \mathrm{~d} \omega
$$

and consequently

$$
\sup _{\|\hat{f}\|_{1} \leq 1} \int_{-\infty}^{\infty}\left(\hat{h}_{1}(\omega)-\hat{h}_{2}(\omega)\right) \hat{f}(\omega) \mathrm{e}^{i \omega t} \mathrm{~d} \omega=0
$$

for all $f \in \mathcal{W}$. Since

$$
\sup _{\|\hat{f}\|_{1} \leq 1} \int_{-\infty}^{\infty}\left(\hat{h}_{1}(\omega)-\hat{h}_{2}(\omega)\right) \hat{f}(\omega) \mathrm{e}^{i \omega t} \mathrm{~d} \omega=\left\|\hat{h}_{1}-\hat{h}_{2}\right\|_{\infty},
$$

we have $\left\|\hat{h}_{1}-\hat{h}_{2}\right\|_{\infty}=0$, which implies that $\hat{h}_{1}=\hat{h}_{2}$.

\section{B Supplement to the Proof of Theorem 4}

Part 1:

$$
\alpha(r)=\sum_{l=1}^{r-1} \frac{1}{l^{2}} \sum_{k=-M_{r}}^{M_{r}} \frac{\sin \left(\frac{k \pi}{a M_{l}}\right)}{\frac{k \pi}{a M_{l}}} \tilde{\phi}_{1}\left(\frac{M_{r}+1-k}{a}\right),
$$

The second sum in (27) can be split into the two parts

$$
\sum_{k=-M_{r}}^{M_{r}} \frac{\sin \left(\frac{k \pi}{a M_{l}}\right)}{\frac{k \pi}{a M_{l}}} \frac{1}{a \pi\left(\frac{M_{r}+1-k}{a}\right)}
$$

and

$$
\sum_{k=-M_{r}}^{M_{r}} \frac{\sin \left(\frac{k \pi}{a M_{l}}\right)}{\frac{k \pi}{a M_{l}}} \frac{2\left(\sin \left(\frac{\pi\left(M_{r}+1-k\right)}{2 a}\right)-\sin \left(\frac{\pi\left(M_{r}+1-k\right)}{a}\right)\right)}{a \pi^{2}\left(\frac{M_{r}+1-k}{a}\right)^{2}}
$$

after (19) is inserted. Obviously, the modulus of (29) can be bounded above by

$$
\frac{4 a}{\pi^{2}} \sum_{k=-M_{r}}^{M_{r}} \frac{1}{\left(M_{r}+1-k\right)^{2}}=\frac{4 a}{\pi^{2}} \sum_{k=1}^{2 M_{r}+1} \frac{1}{k^{2}}<\frac{2 a}{3} .
$$


In order to upper bound the modulus of (28) we rearrange the expression

$$
\begin{aligned}
\left|\sum_{k=-M_{r}}^{M_{r}} \frac{\sin \left(\frac{k \pi}{a M_{l}}\right)}{\frac{k \pi}{a M_{l}}} \frac{1}{a \pi\left(\frac{M_{r}+1-k}{a}\right)}\right| \\
=\frac{1}{\pi\left(M_{r}+1\right)}+\frac{a M_{l}}{\pi^{2}} \mid \sum_{k=1}^{M_{r}} \underbrace{\sin \left(\frac{k \pi}{a M_{l}}\right)}_{=c_{k}} \underbrace{\frac{1}{k\left(M_{r}+1-k\right)}}_{=d_{k}^{-}} \\
+\sum_{k=1}^{M_{r}} \underbrace{\sin \left(\frac{k \pi}{a M_{l}}\right)}_{=c_{k}} \underbrace{\frac{1}{k\left(M_{r}+1+k\right)}}_{=d_{k}^{+}}
\end{aligned}
$$

and do summation by parts. Let $C_{k}=\sum_{n=1}^{k} c_{l},|k| \leq M_{r}$. Then

$$
\begin{aligned}
\left|C_{k}\right| & =\left|\sum_{n=1}^{k} \sin \left(\frac{n \pi}{a M_{l}}\right)\right|=\frac{1}{2}\left|\sum_{n=1}^{k} \mathrm{e}^{i n \pi /\left(a M_{l}\right)}-\mathrm{e}^{-i n \pi /\left(a M_{l}\right)}\right| \\
& \leq \frac{1}{2}\left|\frac{\mathrm{e}^{i \pi(k+1) /\left(a M_{l}\right)}-\mathrm{e}^{-i \pi /\left(a M_{l}\right)}\left|+\frac{1}{2}\right| \frac{\mathrm{e}^{-i \pi(k+1) /\left(a M_{l}\right)}-\mathrm{e}^{i \pi /\left(a M_{l}\right)}}{\mathrm{e}^{i \pi /\left(a M_{l}\right)}-1} \mid}{\mathrm{e}^{-i \pi /\left(a M_{l}\right)}-1}\right| \\
& \leq \frac{1}{\left|\mathrm{e}^{i \pi /\left(a M_{l}\right)}-1\right|}+\frac{1}{\left|\mathrm{e}^{-i \pi /\left(a M_{l}\right)}-1\right|}=\frac{1}{\sin \left(\frac{\pi}{2 a M_{l}}\right)}
\end{aligned}
$$

and

$$
\begin{aligned}
\left|\sum_{k=1}^{M_{r}} c_{k} d_{k}\right| & \leq\left|C_{M_{r}} d_{M_{r}}\right|+\sum_{k=1}^{M_{r}-1}\left|C_{k}\right|\left|d_{k}-d_{k+1}\right| \\
& \leq \frac{1}{\sin \left(\frac{\pi}{2 a M_{l}}\right)}\left(d_{M_{r}}+\sum_{k=1}^{M_{r}-1}\left|d_{k}\right|+\sum_{k=1}^{M_{r}-1}\left|d_{k+1}\right|\right) .
\end{aligned}
$$

Since

$$
\begin{aligned}
\sum_{k=1}^{M_{r}-1}\left|d_{k}^{-}\right| & =\sum_{k=1}^{M_{r}-1} \frac{1}{k\left(M_{r}+1-k\right)}=\frac{1}{M_{r}+1} \sum_{k=1}^{M_{r}-1} \frac{1}{k}+\frac{1}{M_{r}+1-k} \\
& =\frac{1}{M_{r}+1}\left(1+\sum_{k=2}^{M_{r}-1} \frac{1}{k}+\sum_{k=2}^{M_{r}} \frac{1}{k}\right) \\
& <\frac{1}{M_{r}+1}\left(1+\log \left(M_{r}-1\right)+\log \left(M_{r}\right)\right)<\frac{1+2 \log \left(M_{r}\right)}{M_{r}}
\end{aligned}
$$




$$
\begin{aligned}
\sum_{k=1}^{M_{r}-1}\left|d_{k+1}^{-}\right| & =\sum_{k=1}^{M_{r}-1} \frac{1}{(k+1)\left(M_{r}-k\right)}=\frac{1}{M_{r}+1} \sum_{k=1}^{M_{r}-1} \frac{1}{k+1}+\frac{1}{M_{r}-k} \\
& =\frac{1}{M_{r}+1}\left(1+\sum_{k=2}^{M_{r}-1} \frac{1}{k}+\sum_{k=2}^{M_{r}} \frac{1}{k}\right) \\
& <\frac{1}{M_{r}+1}\left(1+\log \left(M_{r}-1\right)+\log \left(M_{r}\right)\right)<\frac{1+2 \log \left(M_{r}\right)}{M_{r}} \\
\sum_{k=1}^{M_{r}-1}\left|d_{k}^{+}\right| & =\sum_{k=1}^{M_{r}-1} \frac{1}{k\left(M_{r}+1+k\right)}=\frac{1}{M_{r}+1} \sum_{k=1}^{M_{r}-1} \frac{1}{k}-\frac{1}{M_{r}+1+k} \\
& <\frac{1}{M_{r}+1}\left(1+\log \left(M_{r}-1\right)\right)<\frac{1+\log \left(M_{r}\right)}{M_{r}}
\end{aligned}
$$

and

$$
\begin{aligned}
\sum_{k=1}^{M_{r}-1}\left|d_{k+1}^{+}\right| & =\sum_{k=1}^{M_{r}-1} \frac{1}{(k+1)\left(M_{r}+2+k\right)}=\frac{1}{M_{r}+1} \sum_{k=1}^{M_{r}-1} \frac{1}{k+1}-\frac{1}{M_{r}+2+k} \\
& <\frac{1}{M_{r}+1} \log \left(M_{r}\right)<\frac{1+\log \left(M_{r}\right)}{M_{r}}
\end{aligned}
$$

we obtain

$$
\begin{aligned}
& \left|\sum_{k=-M_{r}}^{M_{r}} \frac{\sin \left(\frac{k \pi}{a M_{l}}\right)}{\frac{k \pi}{a M_{l}}} \frac{1}{a \pi\left(\frac{M_{r}+1-k}{a}\right)}\right| \\
& <\frac{1}{\pi\left(M_{r}+1\right)}+\frac{a M_{l}}{\pi^{2} \sin \left(\frac{\pi}{2 a M_{l}}\right)}\left(\frac{5+6 \log \left(M_{r}\right)}{M_{r}}+\frac{1}{M_{r}\left(2 M_{r}+1\right)}\right) \\
& <\frac{a^{2} M_{l}^{2}}{\pi\left(M_{r}+1\right)}\left(\frac{5+6 \log \left(M_{r}\right)}{\pi^{2}}+\frac{1}{M_{r}\left(2 M_{r}+1\right)}\right),
\end{aligned}
$$

because $\sin (x) \geq 2 x / \pi$ for all $0 \leq x \leq \pi / 2$.

Inserting (30) and (31) into (27) gives

$$
\begin{aligned}
|\alpha(r)| & \leq \sum_{l=1}^{r-1} \frac{1}{l^{2}}\left(\frac{2 a}{3}+\frac{1}{\pi\left(M_{r}+1\right)}+\frac{a^{2} M_{l}^{2}}{\pi^{2} M_{r}}\left(5+6 \log \left(M_{r}\right)+\frac{1}{2 M_{r}+1}\right)\right) \\
& \leq\left(\frac{2 a}{3}+\frac{1}{\pi\left(M_{r}+1\right)}+\frac{a^{2} M_{r-1}^{2}}{\pi^{2} M_{r}}\left(5+6 \log \left(M_{r}\right)+\frac{1}{2 M_{r}+1}\right)\right) \frac{\pi^{2}}{6} .
\end{aligned}
$$

Moreover, by the construction of the sequence $\left(M_{r}\right)_{r \in \mathbb{Z}}$ we have:

$$
M_{r-1}^{2} \log \left(M_{r}\right) / M_{r}<1,
$$

and therefore $\lim _{r \rightarrow \infty}|\alpha(r)| \leq C_{12}$ with some constant $C_{12}<\infty$. 